Research Article

\title{
Modeling and 1 : 1 Internal Resonance Analysis of Cable-Stayed Shallow Arches
}

\author{
Jiangen Lv $\mathbb{D}^{1},{ }^{1}$ Zhicheng Yang, ${ }^{1}$ Xuebin Chen, ${ }^{1}$ Quanke Wu, ${ }^{1}$ and Xiaoxia Zeng ${ }^{2}$ \\ ${ }^{1}$ College of Urban and Rural Construction, Zhongkai University of Agriculture and Engineering, Guangzhou 510225, China \\ ${ }^{2}$ School of Civil Engineering and Transportation, South China University of Technology, Guangzhou 510640, China \\ Correspondence should be addressed to Jiangen Lv; lvjiangen77@163.com
}

Received 31 October 2019; Revised 23 July 2020; Accepted 11 August 2020; Published 26 August 2020

Academic Editor: Silvio L. T. de Souza

Copyright (c) 2020 Jiangen Lv et al. This is an open access article distributed under the Creative Commons Attribution License, which permits unrestricted use, distribution, and reproduction in any medium, provided the original work is properly cited.

In this paper, an analytical model of a cable-stayed shallow arch is developed in order to investigate the $1: 1$ internal resonance between modes of a cable and a shallow arch. Integrodifferential equations with quadratic and cubic nonlinearities are used to model the in-plane motion of a simple cable-stayed shallow arch. Nonlinear dynamic responses of a cable-stayed shallow arch subjected to external excitations with simultaneous $1: 1$ internal resonances are investigated. Firstly, the Galerkin method is used to discretize the governing nonlinear integral-partial-differential equations. Secondly, the multiple scales method (MSM) is used to derive the modulation equations of the system under external excitation of the shallow arch. Thirdly, the equilibrium, the periodic, and the chaotic solutions of the modulation equations are also analyzed in detail. The frequency- and force-response curves are obtained by using the Newton-Raphson method in conjunction with the pseudoarclength path-following algorithm. The cascades of period-doubling bifurcations leading to chaos are obtained by applying numerical simulations. Finally, the effects of key parameters on the responses are examined, such as initial tension, inclined angle of the cable, and rise and inclined angle of shallow arch. The comprehensive numerical results and research findings will provide essential information for the safety evaluation of cable-supported structures that have widely been used in civil engineering.

\section{Introduction}

Cable-supported structures have been widely used in civil engineering for its light weight, flexibleness, and low fundamental damping $[1,2]$. Because of their large amplitude vibrations under environmental loads, these structures are susceptible to loss of serviceability or even failure. It is quite crucial to understand the dynamic characteristics of cablesupported structures under environmental loads $[3,4]$.

According to previous studies, the support motion effect on cable-supported systems is complicated and thus has been simplified as a boundary excitation in the analysis process. Benedettini et al. [5] established a four-degree-offreedom discrete cable model for modal couplings under vertical/out-of-plane support motions. Guo et al. [6] investigated cable's mode interactions under vertical support motions by boundary resonant modulation. Lee et al. [7] studied free vibration of a rotating curved beam with elastically restrained roots. The two-to-one internal resonance between nonlinear normal modes of an elastically constrained shallow arch is examined [8]. In these cases, the assumption would be reasonable if the mass of the support is much larger than that of cable. Moreover, several studies have been carried out on the dynamic characteristic of cablestayed beam. Abdel-Ghaffar et al. $[3,9,10]$ presented the refined finite element models for cable-stayed bridge dynamics to show the importance of nonlinear dynamical analysis and of cable beam interaction. Fujino et al. [11-13] proposed to study cable beam interactions using a Ritz-type analytical model based on the test function representative of global and local modes and through an experimental set-up of a cable-stayed beam. Gattulli et al. [14] presented a parametric investigation of both linear and nonlinear characteristics of a simple cable-stayed beam and explored the interaction mechanisms of cable beams by means of numerical simulations. In particular, in order to completely 
explore the interaction mechanisms, they presented a precise comparison between the findings obtained by an analytical model for a simple geometrically nonlinear cable-stayed beam [15] and those obtained by both finite element analysis and experiments on a physical model [16], and they also investigated the localization and veering in the dynamics of cable-stayed bridges by a cable-stayed beam model [17]. Wei et al. [18] studied bifurcation and chaos of a cable beamcoupled system under simultaneous internal and external resonances. Kang et al. [19] numerically investigated the nonlinear dynamic response of the stay cable in cable-stayed beam subjected to parametrical and forced excitations. Generally speaking, the nonlinear dynamic problem has been solved by approximate method in much literature, and some researchers validated the approximate results by numerical simulations and/or experimental method. The static configuration of a beam was considered, and the nonlinear modal properties of cable-stayed beam with the direct approach and discretization approach were investigated on the basis of the exact mode shapes of cable-stayed beam [20]. Srinil and Rega [21] presented space-time numerical simulation and validation of analytical predictions for the finiteamplitude forced dynamics of suspended cables. Luongo and Zulli [22] validated the strongly modulated response of the string and the NES by the Galerkin model.

Many of the studies mentioned above are focused on the dynamic behavior of cable-stayed beams, and little work has been done on dynamic studies of cable-stayed shallow arches. With the development of long-span structure, cablestayed shallow arches are gradually widely used in civil engineering, especially in the arch bridge construction process and other long-span building structure. The main advantages of the cable-stayed shallow arch system are that this system has a good use of the compressive strength of the arch and the tensile strength of the cable. However, the structures of the system thus become complex. In spite of this, the cable-stayed shallow arch system has also attracted the attention of many researchers. Ju and Guo [23] studied the in-plane elastic buckling behavior of a cable-stayed shallow arch structure. Ai et al. [24] proposed a practical calculation method of the critical lateral flexure load of cable-stayed shallow arch systems, and the effect of the stay cable on the lateral stability of a cable-arch system was discussed. Zhao and Kang $[25,26]$ investigated the in-plane and out-of-plane free vibration of a cable-supported arch structure and its inherent properties. Most researches on dynamic characteristics of cable-stayed arches did not take into account the nonlinearity. There are only a few studies that considered geometrical nonlinearity: Lv et al. [27] established the governing equations of cable-stayed arch structures, and the possible internal resonance of cablestayed arch structures was also investigated. Kang et al. [28] investigated the in-plane $1: 1: 1$ internal resonance between three first modes of the shallow arch and two cables under both external primary and subharmonic resonance.

In cable-stayed beam structures, two different modal shapes can be basically identified: some modal shapes mainly involve the dynamic beam deflection, while the cable seems to be quasistatically dragged by the beam tip; in other modes, the deflection is extremely localized in the cable domain, with negligible beam participation in the modal shape [17]. Similarly, the cable-dominant vibration can be classified as local modes, while the arch-dominant vibration may be named global modes (since the arch ideally represents the main structure of a cable-stayed arch structure). For a specific cable-supported arch structure, there may be three main resonance mechanisms: $1: 1$ internal resonance, twoto-one internal resonance, and one-to-two internal resonance. Hence, this paper is devoted to the investigation of the $1: 1$ internal resonance of cable-stayed shallow arches. The governing equation for the cable-stayed shallow arch is derived by using Hamilton's principle and solved by the Galerkin method and multiple scales method. The effects of initial tension, inclined angle of the cable and arch, and arch geometry on the 1:1 internal resonance of the cable-stayed shallow arch are studied comprehensively.

\section{Modeling and Equations of Motion}

A cable-stayed shallow arch is used as the supporting member in the arch bridge system (Figure 1), and its first construction stage model containing a shallow arch and a single cable is shown in Figure 2. The static and dynamic models used in following analyses for the cable-stayed shallow arch are shown in Figure 3. The cable and shallow arch are homogeneous and rigidly connected at the end. Shear inertia and rotary deformation of the shallow arch are neglected. The rise $d_{g}$ to length $l_{g}$ of arch is small (i.e., $d_{g} / l_{g} \leq 1 / 10$, namely, shallow arch); similarly, the sag $d_{\mathrm{c}}$ to length $l_{c}$ of cable is small (i.e., $d_{c} / l_{c} \leq 1 / 10$ ). The shallow arch is subjected to a harmonic load, which can be expressed in the form $P(\bar{x}, t)=G(\bar{x}) \cos (\bar{\Omega} t), G(\bar{x})$ is the amplitude of the transverse excitation applied to the shallow arch, and $\bar{\Omega}$ is the excitation frequency. $u_{c}$ and $v_{c}$ are the longitudinal and transverse displacements of the cable, respectively, and $l_{c}$ its span length, $d_{c}$ its sag, and $\theta_{c}$ its inclined angle; $u_{g}$ and $v_{g}$ are the longitudinal and transverse displacements of shallow arch, respectively, and $l_{g}$ its span lengths, $d_{g}$ its rise, and $\theta_{g}$ its inclined angle.

To ensure continuity at connection point between the cable and the shallow arch and projecting along the $v_{c}$ and $v_{g}$ directions, the following equations are obtained:

$$
\begin{aligned}
& u_{g}\left(l_{g}, t\right)=\frac{-v_{c}\left(l_{c}, t\right)-v_{g}\left(l_{g}, t\right) \cos \left(\theta_{g}+\theta_{c}\right)}{\sin \left(\theta_{g}+\theta_{c}\right)}, \\
& u_{c}\left(l_{c}, t\right)=\frac{-v_{g}\left(l_{g}, t\right)-v_{c}\left(l_{c}, t\right) \cos \left(\theta_{g}+\theta_{c}\right)}{\sin \left(\theta_{g}+\theta_{c}\right)},
\end{aligned}
$$

where $m_{c}$ denotes the mass per unit length of the cable, $c_{c}$ its damping coefficient, $y_{0}$ its static displacement, $E_{c} A_{c}$ its axial stiffness, and $H$ its initial tension. $m_{g}$ denotes mass per unit length of the shallow arch, $c_{g}$ its damping coefficients, $E_{g} A_{g}$ its axial stiffness, $\bar{y}_{0}$ its static displacement, $E_{g} I_{g}$ its flexural stiffness, and $N$ its initial axial force. Equations of motion, which govern the transverse vibrations, are obtained by the classical extended Hamilton's principle: 


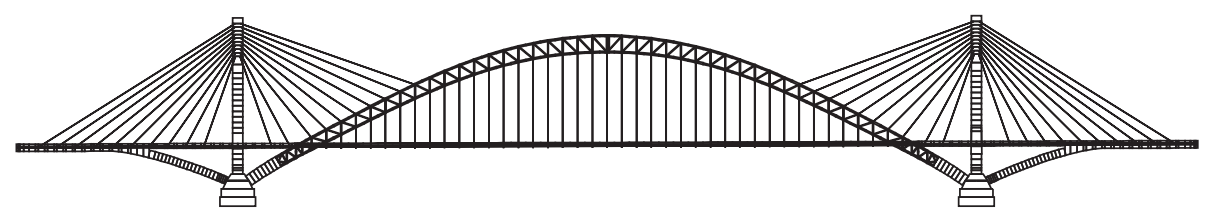

FIgURE 1: Lateral view of a cable-stayed shallow arch bridge.

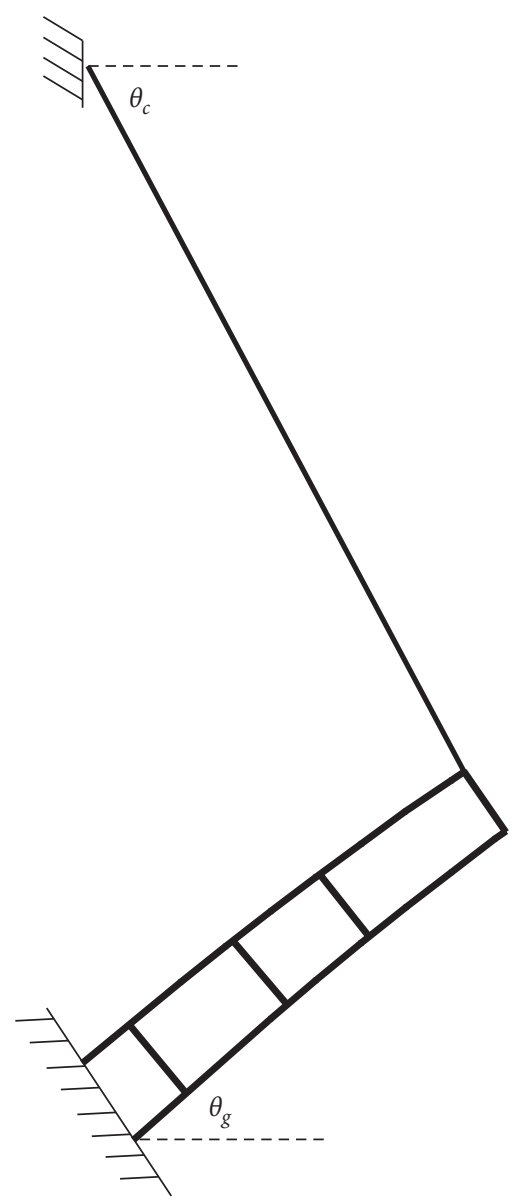

Figure 2: The first construction stage.

$$
\begin{gathered}
m_{c} \frac{\partial^{2} v_{c}}{\partial t^{2}}+c_{c} \frac{\partial v_{c}}{\partial t}-H \frac{\partial^{2} v_{c}}{\partial x^{2}}-E_{c} A_{c}\left(\frac{\partial^{2} y_{0}}{\partial x^{2}}+\frac{\partial^{2} v_{c}}{\partial x^{2}}\right) e_{c}(t)=0 \\
m_{g} \frac{\partial^{2} v_{g}}{\partial t^{2}}+c_{g} \frac{\partial v_{g}}{\partial t}-N \frac{\partial^{2} v_{g}}{\partial \bar{x}^{2}}+E_{g} I_{g} \frac{\partial^{4} v_{g}}{\partial \bar{x}^{4}}-E_{g} A_{g}\left(\frac{\partial^{2} \bar{y}_{0}}{\partial \bar{x}^{2}}+\frac{\partial^{2} v_{g}}{\partial \bar{x}^{2}}\right) e_{g}(t)=G(\bar{x}) \cos (\bar{\Omega} t)
\end{gathered}
$$

where

$$
\begin{aligned}
& e_{c}(t)=-\frac{v_{g}\left(l_{g}, t\right)+v_{c}\left(l_{c}, t\right) \cos \left(\theta_{g}+\theta_{c}\right)}{l_{c} \sin \left(\theta_{g}+\theta_{c}\right)}+\frac{1}{l_{c}} \int_{0}^{l_{c}}\left[\frac{\mathrm{d} y_{0}}{\mathrm{~d} x} \frac{\mathrm{d} v_{c}}{\mathrm{~d} x}+\frac{1}{2}\left(\frac{\mathrm{d} v_{c}}{\mathrm{~d} x}\right)^{2}\right] \mathrm{d} x, \\
& e_{g}(t)=-\frac{v_{c}\left(l_{c}, t\right)+v_{g}\left(l_{g}, t\right) \cos \left(\theta_{g}+\theta_{c}\right)}{l_{g} \sin \left(\theta_{g}+\theta_{c}\right)}+\frac{1}{2 l_{g}} \int_{0}^{l_{g}}\left[\left(\frac{\partial v_{g}}{\partial \bar{x}}+\frac{\partial \bar{y}_{0}}{\partial \bar{x}}\right)^{2}-\left(\frac{\partial \bar{y}_{0}}{\partial \bar{x}}\right)^{2}\right] \mathrm{d} \bar{x} .
\end{aligned}
$$




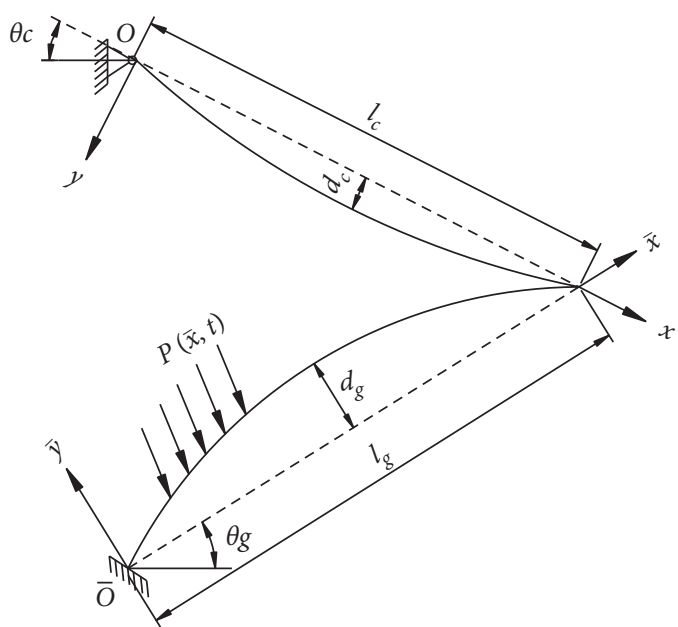

(a)

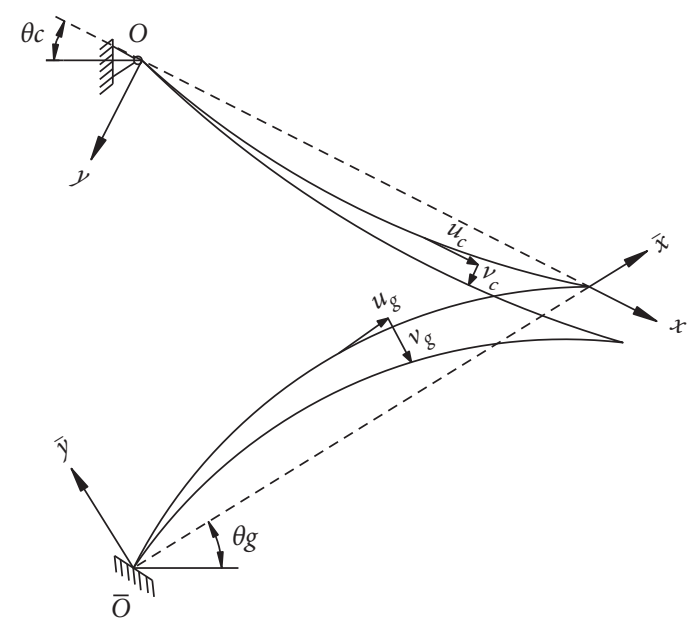

(b)

Figure 3: Configuration of a cable-stayed shallow arch: (a) static configuration; (b) dynamic configuration.

As can be seen from equations (2) and (3), compared with a cable-stayed beam system, equations of the cablestayed shallow arch system are more complex. The cable's equation of motion of the cable-stayed beam system is in agreement with that of the cable-stayed shallow arch system, while the shallow arch's equation motion of the cable-stayed shallow arch is different from that of beam in the cable-

$$
\begin{aligned}
& v_{c}(0, t)=v_{g}(0, t)=0, \\
& v_{g}^{\prime}(0, t)=0, \\
& v_{g}^{\prime \prime}(0, t)=0,
\end{aligned}
$$

$$
\left\{H v_{c}^{\prime}\left(l_{c}, t\right)+E_{c} A_{c} e_{c}(t) \cdot\left[v_{c}^{\prime}\left(l_{c}, t\right)+y_{0}^{\prime}\left(l_{c}, t\right)\right]\right\} \cdot \cos \left(\theta_{c}+\theta_{g}\right)+E_{c} A_{c} e_{c}(t) \sin \left(\theta_{c}+\theta_{g}\right)=E_{g} I_{g} v_{g}^{\prime \prime}\left(l_{g}, t\right) .
$$

As shown in Figure 4, the cable is anchored at the junction $S$; $S^{\prime}$ is the deformed position of $S$; $u_{c}=u_{c}\left(l_{c}, t\right)$; $v_{c}=v_{c}\left(l_{c}, t\right) ; v_{g}=v_{g}\left(l_{g}, t\right) ; Q$ and $Q^{\prime}$ are the shear forces acting on the left and right of the junction, respectively; $M$ and $M^{\prime}$ are the bending moment acting on the left and right of the junction, separately; and $T_{c x}=E_{c} A_{c} e_{c}(t)$ and $T_{c y}=$ $H v_{c}^{\prime}\left(l_{c}, t\right)+E_{c} A_{c} e_{c}(t) \cdot\left[v_{c}^{\prime}\left(l_{c}, t\right)+y_{0}^{\prime}\left(l_{c}, t\right)\right]$ are the components of the tension of cable in the longitudinal and transverse directions, respectively.

2.1. Galerkin Discretization Procedure. Using the discretization approach, Lacarbonara et al. [30] have obtained the general modulation equations of the displacement in distributed-parameter systems. Similarly, following the methodologies in [30], the discretization approach is used to obtain the modulation equations governing the nonlinear dynamic behavior of cable-stayed shallow arches with $1: 1$ internal resonances. Then, the in-plane transverse stayed beam because of the geometric curvature of shallow arch, and it is consistent with that equation in [29]. As a result of these different from the cable-stayed beam, it is very necessary to study the nonlinear dynamic characteristics of the cable-stayed shallow arch structure.

The geometric and mechanical boundary conditions at the junction can be written as follows: displacements $v_{c}$ and $v_{g}$ are approximately expressed in the following form:

$$
\begin{aligned}
& v_{c}(x, t)=\sum_{i=1}^{\infty} \varphi_{i}(x) q_{i}(t), \\
& v_{g}(\bar{x}, t)=\sum_{j=1}^{\infty} \phi_{j}(\bar{x}) \bar{q}_{j}(t),
\end{aligned}
$$

where $\varphi_{i}(x)$ and $\phi_{j}(\bar{x})$ are the mode shape functions of the cable and shallow arch, respectively, with $i=1,2,3, \ldots$ referring to the natural modes of the linearized problem (e.g., Figure 5). $q_{i}(t)$ and $\bar{q}_{i}(t)$ are the generalized coordinates of the cable and shallow arch.

Equations (6) and (7) are substituted into equations (2) and (3) and integrated where required, and the Galerkin method is used to obtain a nonlinear model with the following system of differential equations for the cable and shallow arch motions: 


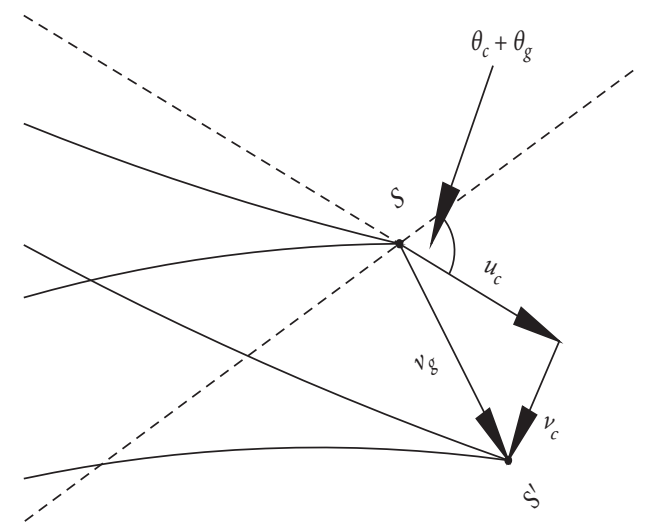

(a)

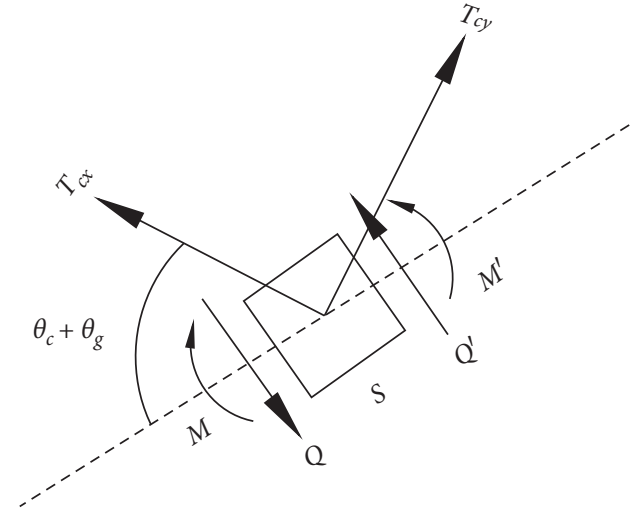

(b)

FIgURE 4: Schematic of the (a) geometric and (b) mechanical boundary conditions at the junction.

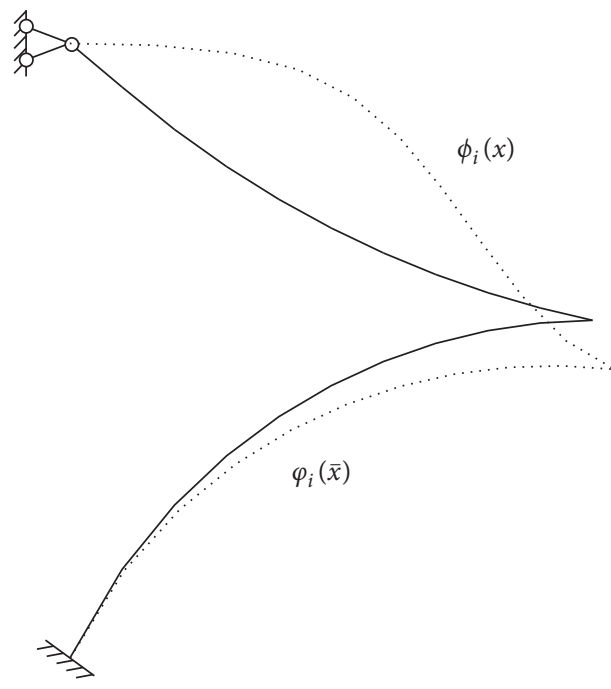

(a)

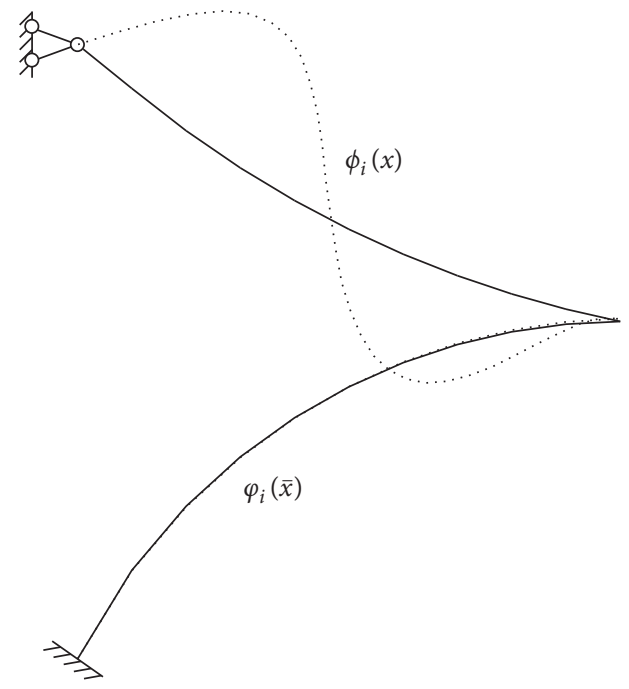

(b)

Figure 5: Modal shapes of the cable-stayed shallow arch: (a) global mode; (b) local mode.

$$
\begin{gathered}
\ddot{q}_{i}+2 \mu_{1, i} \dot{q}_{i}+\omega_{1, i}^{2} q_{i}+\sum_{j=1, j \neq i}^{\infty} \alpha_{1, i j} q_{j}+\sum_{j=1}^{\infty} \sum_{k=1}^{\infty} \sum_{l=1}^{\infty}\left(\alpha_{3, i j} \bar{q}_{j}+\beta_{1, j k} q_{j} \bar{q}_{k}+\beta_{3, i j k} q_{j} q_{k}+\gamma_{1, i j k l} q_{j} q_{k} q_{l}\right)=0, \\
\ddot{\bar{q}}_{j}+2 \mu_{2, j} \dot{\bar{q}}_{j}+\omega_{2, j}^{2} \bar{q}_{j}+\sum_{i=1, i \neq j}^{\infty} \alpha_{2, j i} \bar{q}_{i}+\sum_{i=1}^{\infty} \sum_{k=1}^{\infty} \sum_{l=1}^{\infty}\left(\alpha_{4, j i} q_{i}+\beta_{2, j i k} \bar{q}_{i} q_{k}+\beta_{4, j i k} \bar{q}_{i} \bar{q}_{k}+\gamma_{2, j i k l} \bar{q}_{i} \bar{q}_{k} \bar{q}_{l}\right)=g_{j} \cos \left(\bar{\Omega}_{j} t\right),
\end{gathered}
$$

where the coefficients are defined in the Appendix, $g_{j}$ is the amplitude of the transverse excitation applied to the shallow arch, and $\bar{\Omega}_{j}$ is the excitation frequency.

2.2. Perturbation Analysis. The multiple scale method [31] is used to seek a first-order uniform expansion of the solution of equations (8) and (9) of the following form:

$$
\begin{aligned}
& q_{n}\left(T_{0}, T_{1}, \varepsilon\right)=q_{0, n}\left(T_{0}, T_{1}\right)+\varepsilon q_{1, n}\left(T_{0}, T_{1}\right), \\
& \bar{q}_{n}\left(T_{0}, T_{1}, \varepsilon\right)=\bar{q}_{0, n}\left(T_{0}, T_{1}\right)+\varepsilon \bar{q}_{1, n}\left(T_{0}, T_{1}\right),
\end{aligned}
$$

where $T_{i}=\varepsilon^{i} t(i=0,1) . T_{0}$ is the fast time scale associated with changes occurring at $\omega_{1, i}, \omega_{2, j}$, and $\Omega_{j}$, and $T_{1}$ is the slow time scale associated with modulations in the amplitudes and phases caused by nonlinearity, damping, and resonance. In terms of $T_{0}$ and $T_{1}$, the time derivatives become 


$$
\begin{aligned}
\frac{\mathrm{d}}{\mathrm{d} t} & =\frac{\partial}{\partial T_{0}}+\varepsilon \frac{\partial}{\partial T_{1}}=D_{0}+\varepsilon D_{1}, \\
\frac{\mathrm{d}^{2}}{0 t^{2}} & =\left(\frac{\partial}{\partial T_{0}}+\varepsilon \frac{\partial}{\partial T_{1}}\right)^{2}=D_{0}^{2}+2 \varepsilon D_{0} D_{1}+\varepsilon^{2} D_{1}^{2}
\end{aligned}
$$

where $D_{i}=\partial / \partial T_{i}(i=0,1)$. Substituting them into equations (8) and (9) and then equating coefficients of the same power of $\varepsilon$, we obtain the following differential equations:

$$
\begin{gathered}
\varepsilon 0: D_{0}^{2} q_{0, n}+\omega_{1, n}^{2} q_{0, n}=0, \\
D_{0}^{2} \bar{q}_{0, n}+\omega_{2, n}^{2} \bar{q}_{0, n}=0
\end{gathered}
$$

$\varepsilon 1: D_{0}^{2} q_{1, n}+\omega_{1, n}^{2} q_{1, n}=-2 D_{0} D_{1} q_{0, n}-2 \mu_{1, n} D_{0} q_{0, n}-\sum_{m=1, m \neq n}^{\infty} \alpha_{1, m n} q_{0, m}-\sum_{m, p, q=1}^{\infty}\left(\alpha_{3, m n} \bar{q}_{0, m}+\beta_{1, m p n} q_{0, m} \bar{q}_{0, p}+\beta_{3, m p n} q_{0, m} q_{0, p}\right.$ $\left.+\gamma_{1, m p q n} q_{0, m} q_{0, p} q_{0, q}\right)$

$$
\begin{aligned}
D_{0}^{2} \bar{q}_{1, n}+\omega_{2, n}^{2} \bar{q}_{1, n}= & -2 D_{0} D_{1} \bar{q}_{0, n}-2 \mu_{2, n} D_{0} \bar{q}_{0, n}-\sum_{m=1, m \neq n}^{\infty} \alpha_{2, m n} \bar{q}_{0, m}-\sum_{m, p, q=1}^{\infty}\left(\alpha_{4, m n} q_{0, m}+\beta_{2, m p n} \bar{q}_{0, m} q_{0, p}+\beta_{4, m p n} \bar{q}_{0, m} \bar{q}_{0, p}\right. \\
& \left.+\gamma_{2, m p q n} \bar{q}_{0, m} \bar{q}_{0, p} \bar{q}_{0, q}\right)+g_{n} \cos \left(\bar{\Omega}_{n} t\right),
\end{aligned}
$$

The general solution of equations (12) and (13) can be expressed as

$$
\begin{aligned}
& q_{0, n}\left(T_{0}, T_{1}\right)=A_{n}\left(T_{1}\right) \exp \left(i \omega_{1, n} T_{0}\right)+c c, \\
& \bar{q}_{0, n}\left(T_{0}, T_{1}\right)=B_{n}\left(T_{1}\right) \exp \left(i \omega_{2, n} T_{0}\right)+c c,
\end{aligned}
$$

where $A_{n}=1 / 2 a_{n}\left(T_{1}\right) e^{i \theta_{n}\left(T_{1}\right)}$ and $B_{m}=1 / 2 b_{m}\left(T_{1}\right) e^{i \bar{\theta}_{m}\left(T_{1}\right)}$ with $a_{n}, b_{m}$ and $\theta_{n}, \theta_{m}$ denoting the steady-state amplitudes and the phases of motion, respectively. $c c$ denotes a complex conjugate of the preceding term. Substituting equations (16) and (17) into equations (14) and (15), we get

$$
\begin{aligned}
D_{0}^{2} q_{1, n}+\omega_{1, n}^{2} q_{1, n}= & -2 i \omega_{1, n} D_{1} A_{n} e^{i \omega_{1, n} T_{0}}-2 i \omega_{1, n} \mu_{1, n} A_{n} e^{i \omega_{1, n} T_{0}}-\sum_{m=1, m \neq n}^{\infty} \alpha_{1, m n} A_{m} e^{i \omega_{1, m} T_{0}} \\
& -\sum_{m, p, q=1}^{\infty}\left\{\alpha_{3, m n} B_{m} e^{i \omega_{2, m} T_{0}}+\beta_{1, m p n}\left[A_{m} B_{p} e^{i\left(\omega_{1, m}+\omega_{2, p}\right) T_{0}}+A_{m} \bar{B}_{p} e^{\left.i\left(\omega_{1, m}-\omega_{2, p}\right) T_{0}\right]}\right.\right. \\
& +\beta_{3, m p n}\left[A_{m} A_{p} e^{i\left(\omega_{1, m}+\omega_{1, p}\right) T_{0}}+A_{m} \bar{A}_{p} e^{i\left(\omega_{1, m}-\omega_{1, p}\right) T_{0}}\right]+\gamma_{1, m p q n}\left[A_{m} A_{p} A_{q} e^{i\left(\omega_{1, m}+\omega_{1, p}+\omega_{1, q}\right) T_{0}}\right. \\
& \left.\left.+A_{m} \bar{A}_{p} A_{q} e^{i\left(\omega_{1, m}-\omega_{1, p}+\omega_{1, q}\right) T_{0}}+\bar{A}_{m} A_{p} A_{q} e^{i\left(\omega_{1, p}+\omega_{1, q}-\omega_{1, m}\right) T_{0}}+\bar{A}_{m} \bar{A}_{p} A_{q} e^{i\left(\omega_{1, q}-\omega_{1, p}-\omega_{1, m}\right) T_{0}}\right]\right\}+c c, \\
D_{0}^{2} \bar{q}_{1, n}+\omega_{2, n}^{2} \bar{q}_{1, n}= & -2 i \omega_{2, n} D_{1} B_{n} e^{i \omega_{2, n} T_{0}}-2 i \omega_{2, n} \mu_{2, n} B_{n} e^{i \omega_{2, n} T_{0}}-\sum_{m=1, m \neq n}^{\infty} \alpha_{2, m n} B_{m} e^{i \omega_{2, m} T_{0}} \\
& -\sum_{m, p, q=1}^{\infty}\left\{\alpha_{4, m n} A_{m} e^{i \omega_{1, m} T_{0}}+\beta_{2, m p n}\left[B_{m} A_{p} e^{i\left(\omega_{2, m}+\omega_{1, p}\right) T_{0}}+B_{m} \bar{A}_{p} e^{i\left(\omega_{2, m}-\omega_{1, p}\right) T_{0}}\right]+\beta_{4, m p n}\left[B_{m} B_{p} e^{i\left(\omega_{2, m}+\omega_{2, p}\right) T_{0}}\right.\right. \\
+ & \left.B_{m} \bar{B}_{p} e^{i\left(\omega_{2, m}-\omega_{2, p}\right) T_{0}}\right]+\gamma_{2, m p q n}\left[B_{m} B_{p} B_{q} e^{i\left(\omega_{2, m}+\omega_{2, p}+\omega_{2, q}\right) T_{0}}+B_{m} \bar{B}_{p} B_{q} e^{i\left(\omega_{2, m}-\omega_{2, p}+\omega_{2, q}\right) T_{0}}\right. \\
+ & \left.\left.\bar{B}_{m} B_{p} B_{q} e^{i\left(\omega_{2, p}+\omega_{2, q}-\omega_{2, m}\right) T_{0}}+\bar{B}_{m} \bar{B}_{p} B_{q} e^{i\left(\omega_{2, q}-\omega_{2, p}-\omega_{2, m}\right) T_{0}}\right]\right\}+\frac{1}{2} g_{n} e^{i \bar{\Omega}_{n} T_{0}}+c c,
\end{aligned}
$$


where the prime indicates the derivative with respect to $T_{1}$. The functions $A_{n}$ and $B_{m}$ can be determined so that the solutions to equations (18) and (19) do not contain secular terms or small-divisor terms caused by resonance.

As shown in equations (18) and (19), there are many types of resonance in cable-stayed shallow arches, such as primary resonance, $1: 1$ internal resonance, $2: 1$ internal resonances, and $1: 2$ internal resonance.

2.3.1:1 Internal Resonance. As the $1: 1$ internal resonance is the most common form of internal resonance in modal interaction, next, the case is analyzed when $1: 1$ internal resonance exists between modes of the cable and shallow arch $\left(\omega_{1, n} \approx \omega_{2, m}\right)$ in the presence of primary resonance of shallow $\operatorname{arch}\left(\bar{\Omega}_{m} \approx \omega_{2, m}\right)$. These resonant relationships, which have been confirmed numerically, can be described as follows:

$$
\bar{\Omega}_{m}=\omega_{2, m}+\varepsilon \sigma_{1} ; \omega_{1, n}=\omega_{2, m}+\varepsilon \sigma_{2},
$$

where $\sigma_{1}$ and $\sigma_{2}$ are two detuning parameters. Substituting equation (20) into equations (18) and (19) and eliminating secular terms yield the following equations:

$$
\begin{gathered}
2 i \omega_{1, n} D_{1} A_{n}+2 i \omega_{1, n} \mu_{1, n} A_{n}+2 \gamma_{1, n m n n} A_{n}^{2} \bar{A}_{n}+\alpha_{3, m n} B_{m} e^{-i \sigma_{2} T_{1}}=0, \\
2 i \omega_{2, m} D_{1} B_{m}+2 i \omega_{2, m} \mu_{2, m} B_{m}+2 \gamma_{2, m m m m} B_{m}^{2} \bar{B}_{m}+\alpha_{4, n m} A_{n} e^{i \sigma_{2} T_{1}}-\frac{1}{2} g_{n} e^{i \sigma_{1} T_{1}}=0 .
\end{gathered}
$$

Substituting $A_{n}$ and $B_{m}$ into equations (21) and (22) and then separating the real and imaginary components give the governing equations for the amplitudes $a_{n}$ and $b_{m}$ and the phases $\gamma_{1}$ and $\gamma_{2}$ :

$$
\begin{aligned}
a_{n}^{\prime} & =-\mu_{1, n} a_{n}-\frac{\alpha_{3 . m n}}{2 \omega_{1, n}} b_{m} \sin \gamma_{1}, \\
a_{n} \theta_{n}^{\prime} & =\frac{\gamma_{1, n m m}}{4 \omega_{1, n}} a_{n}^{3}+\frac{\alpha_{3 . m n}}{2 \omega_{1, n}} b_{m} \cos \gamma_{1}, \\
b_{m}^{\prime} & =-\mu_{2, m} b_{m}+\frac{\alpha_{4 . n m}}{2 \omega_{2, m}} a_{n} \sin \gamma_{1}+\frac{g_{m}}{2 \omega_{2, m}} \sin \gamma_{2}, \\
b_{m} \bar{\theta}_{m}^{\prime} & =\frac{\gamma_{2, m m m m}}{4 \omega_{2, m}} b_{m}^{3}+\frac{\alpha_{4 . n m}}{2 \omega_{2, m}} a_{n} \cos \gamma_{1}-\frac{g_{m}}{2 \omega_{2, m}} \cos \gamma_{2},
\end{aligned}
$$

where $\gamma_{1}=\bar{\theta}_{2}-\theta_{1}-\sigma_{2} T_{1}$ and $\gamma_{2}=\sigma_{1} T_{1}-\bar{\theta}_{2}$. The steadystate solutions correspond to constant solutions, i.e., $a_{n}^{\prime}=b_{m}^{\prime}=\gamma_{1}^{\prime}=\gamma_{2}^{\prime}=0$. Hence, the following set of nonlinear algebraic equations is obtained that is solved numerically to obtain the fixed points of the system:

$$
\begin{gathered}
\mu_{1, n} a_{n}+\frac{\alpha_{3 . m n}}{2 \omega_{1, n}} b_{m} \sin \theta_{1}=0 \\
a_{n}\left(\sigma_{1}-\sigma_{2}\right)-\frac{\gamma_{1, n n n}}{4 \omega_{1, n}} a_{n}^{3}-\frac{\alpha_{3, m n}}{2 \omega_{1, m}} b_{m} \cos \theta_{1}=0 \\
\mu_{2, m} b_{m}-\frac{\alpha_{4 . n m}}{2 \omega_{2, m}} a_{n} \sin \theta_{1}-\frac{g_{m}}{2 \omega_{2, m}} \sin \theta_{2}=0 \\
b_{m} \sigma_{1}-\frac{\gamma_{2, m m m m}}{4 \omega_{2, m}} b_{m}^{3}-\frac{\alpha_{4 . n m}}{2 \omega_{2, m}} a_{n} \cos \theta_{1}+\frac{g_{m}}{2 \omega_{2, m}} \cos \theta_{2}=0 .
\end{gathered}
$$

From equations (24)-(27), we obtain possible solutions besides the trivial solution. Two mode solutions are considered: $a_{n} \neq 0$ and $b_{m} \neq 0$; equations (24) and (25) are squared, the squared results are then added together, and the process is repeated for equations (24)-(27), giving the following frequency-response equations:

$$
\left(\mu_{2, m} b_{m}^{2}+\frac{\mu_{1, n} \alpha_{4, n m} \omega_{1, n}}{\alpha_{3, m n} \omega_{2, m}} a_{n}^{2}\right)^{2}+\left\{\sigma_{2} b_{m}^{2}-\frac{\gamma_{2, m m m m}}{4 \omega_{2, m}} b_{m}^{4}-\frac{\alpha_{4, n m} \omega_{1, n}}{\alpha_{3, m n} \omega_{2, m}}\left[\left(\sigma_{1}-\sigma_{2}\right) a_{n}^{2}-\frac{\gamma_{1, n m n n}}{4 \omega_{1, n}} a_{n}^{4}\right]\right\}^{2}=\frac{g_{m}^{2}}{4 \omega_{2, m}^{2}} b_{m}^{2},
$$

where

$$
b_{m}^{2}=\frac{4 \omega_{1, n}^{2}}{\alpha_{3, m n}^{2}} a_{n}^{2}\left[\mu_{1, n}^{2}+\left(\left(\sigma_{1}-\sigma_{2}\right)-\frac{\gamma_{1, n n m n}}{4 \omega_{1, n}} a_{n}^{2}\right)^{2}\right] .
$$

In order to assess stability of the steady-state solution, it is necessary to obtain the modulation equations in the Cartesian form. Therefore, $A_{n}$ and $B_{m}$ are written in Cartesian form:

$$
\begin{aligned}
& A_{n}=\frac{1}{2}\left(u_{n}+i v_{n}\right) e^{i\left(\sigma_{1}-\sigma_{2}\right) T_{1}}, \\
& B_{m}=\frac{1}{2}\left(u_{m}+i v_{m}\right) e^{i \sigma_{1} T_{1}} .
\end{aligned}
$$

Substituting $A_{n}$ and $B_{m}$ from equation (30) into equations (21) and (22), the following modulation equations in the form of Cartesian form can be obtained by separating the results into real and imaginary parts: 


$$
\begin{gathered}
u_{n}^{\prime}=\left(\sigma_{1}-\sigma_{2}\right) v_{n}-\mu_{1, n} u_{n}-\frac{\gamma_{1, n n n n}}{4 \omega_{1, n}}\left(u_{n}^{2}+v_{n}^{2}\right) v_{n}-\frac{\alpha_{3 . m n}}{2 \omega_{1, n}} v_{m}, \\
v_{n}^{\prime}=-\left(\sigma_{1}-\sigma_{2}\right) u_{n}-\mu_{1, n} v_{n}+\frac{\gamma_{1, n n n n}}{4 \omega_{1, n}}\left(u_{n}^{2}+v_{n}^{2}\right) u_{n}+\frac{\alpha_{3 . m n}}{2 \omega_{1, n}} u_{m}, \\
u_{m}^{\prime}=\sigma_{1} v_{m}-\mu_{2, m} u_{m}-\frac{\gamma_{2, m m m m}}{4 \omega_{2, m}}\left(u_{m}^{2}+v_{m}^{2}\right) v_{m}-\frac{\alpha_{4 . n m}}{2 \omega_{2, m}} v_{n}, \\
v_{m}^{\prime}=-\sigma_{1} u_{m}-\mu_{2, m} v_{m}+\frac{\gamma_{2, m m m m}}{4 \omega_{2, m}}\left(u_{m}^{2}+v_{m}^{2}\right) u_{m}+\frac{\alpha_{4 . n m}}{2 \omega_{2, m}} u_{n} \\
\quad-\frac{g_{m}}{2 \omega_{2, m}} .
\end{gathered}
$$

The periodic motion of cable-stayed shallow arch is mathematically expressed as the steady-state solutions of equations (31)-(33). In order to obtain the steady-state solution, we can assume that $u_{n}^{\prime}=v_{n}^{\prime}=u_{m}^{\prime}=v_{m}^{\prime}=0$ in equations (31)-(33), and the Newton-Raphson method can be applied to solve these four nonlinear equations. After the steady-state $\Gamma_{0}$ is determined $\left(\Gamma_{0}=\left\{u_{n} v_{n} u_{m} v_{m}\right\}^{\mathrm{T}}\right.$, $\mathrm{T}$ is the transpose), the stability of the steady-state solution can then be assessed by applying a classical linearization method [32]. If the steady-state solution is not zero, a new solution $\Gamma=\Gamma_{0}+\Delta \Gamma$ is substituted into these equations. The result is then expanded in a Taylor series about $\Gamma_{0}$, and only the linear terms in the disturbance are retained to yield:

$$
\Delta \dot{\Gamma}=[J] \Delta \Gamma,
$$

where $[\mathrm{J}]$ is called the Jacobian matrix. The stability of the steady-state solution is determined by the eigenvalues of the Jacobian matrix. If all the eigenvalues of Jacobian matrix have a negative real part, the steady-state solution is stable. If the real part of one of the eigenvalues of Jacobian matrix is positive, the steady-state solution is unstable.

\section{Numerical Discussion}

The cable-stayed shallow arch case with following physical parameters is taken from a particular working bridge in China, as shown in Figure 1. The dimensional parameters and material properties of the cable and shallow arch in the first construction stage are listed in Tables 1 and 2, respectively. Moreover, the static equilibrium equation of the shallow arch is approximated by the parabolic function as $\bar{y}_{0}(\bar{x})=-9.532 \times 10^{-4} \bar{x}^{2}+3.954 \times 10^{-2} \bar{x}$ and that of the cable is $y_{0}(x)=-6.973 \times 10^{-5} x^{2}+4.209 \times 10^{-3} x$.

By using the finite element method (FEM), the first ten local and global frequencies of the cable-stayed shallow arch are obtained, as shown in Figure 6. From the figure, the $1: 1$ internal resonance may occur between the local and global modes when $\omega_{1,10} \approx 4.352$ and $\omega_{2,4} \approx 4.415$. Therefore, in this paper, the case of $1: 1$ internal resonance between the 10 th local mode and the 4 th global mode is studied. The solid
TABle 1: Properties of the cable.

\begin{tabular}{lcc}
\hline Item & Notation & Value \\
\hline Mass & $m_{c}$ & $35.325 \mathrm{~kg} / \mathrm{m}$ \\
Extensional rigidity & $E_{c} A_{c}$ & $8.55 \times 105 \mathrm{kN}$ \\
Initial tension & $H$ & $2600 \mathrm{kN}$ \\
Length & $l_{c}$ & $61.951 \mathrm{~m}$ \\
Horizontal angle & $\theta_{c}$ & $51.3^{\circ}$ \\
Damping coefficient & $c_{c}$ & $0.0015 \mathrm{~N} \cdot \mathrm{s} / \mathrm{m}$ \\
\hline
\end{tabular}

TABle 2: Properties of the arch and load.

\begin{tabular}{lcc}
\hline Item & Notation & Value \\
\hline Mass & $m_{g}$ & $2933 \mathrm{~kg} / \mathrm{m}$ \\
Compressive stiffness & $E_{g} A_{g}$ & $7.847 \times 10^{7} \mathrm{kN}$ \\
Length & $l_{g}$ & $41.483 \mathrm{~m}$ \\
Bending stiffness & $E_{g} I_{g}$ & $6.699 \times 10^{6} \mathrm{kN}$ \\
Level elevation & $\theta_{g}$ & $30.7^{\circ}$ \\
Damping coefficient & $c_{g}$ & $0.0015 \mathrm{~N} \cdot \mathrm{s} / \mathrm{m}$ \\
Initial axial force & $N$ & $2412 \mathrm{kN}$ \\
\hline
\end{tabular}

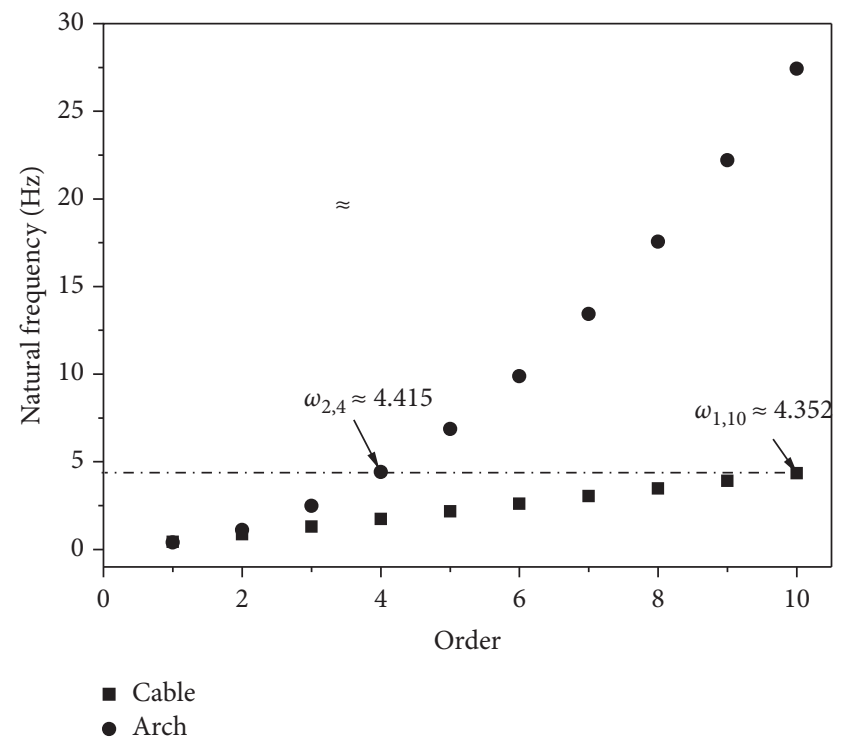

Figure 6: The first ten local and global frequencies of the cablestayed shallow arch.

and dashed lines of the frequency-and force-response curves in next sections indicate the stable and unstable solutions, respectively.

3.1. Equilibrium Solutions, Stability, and Validation. Figure 7 shows variation in the response amplitudes of the cable and shallow arch with the detuning parameter $\sigma_{2}$ when $g_{4}=2.0\left(G_{4}=118.6 \mathrm{kN} / \mathrm{m}\right)$ and $\bar{\Omega}_{4} \approx \omega_{2,4}$, where SNB and $\mathrm{HB}$ represent the saddle-node and Hopf bifurcation, respectively. For the right branch, the composite structure loses its stability due to a Hopf bifurcation at HB1 $\left(\sigma_{2} \approx 0.055\right)$. The system then regains its stability through another Hopf bifurcation at HB2 $\left(\sigma_{2} \approx 0.087\right)$. Due to the effect of internal resonance, the cable that is not directly 


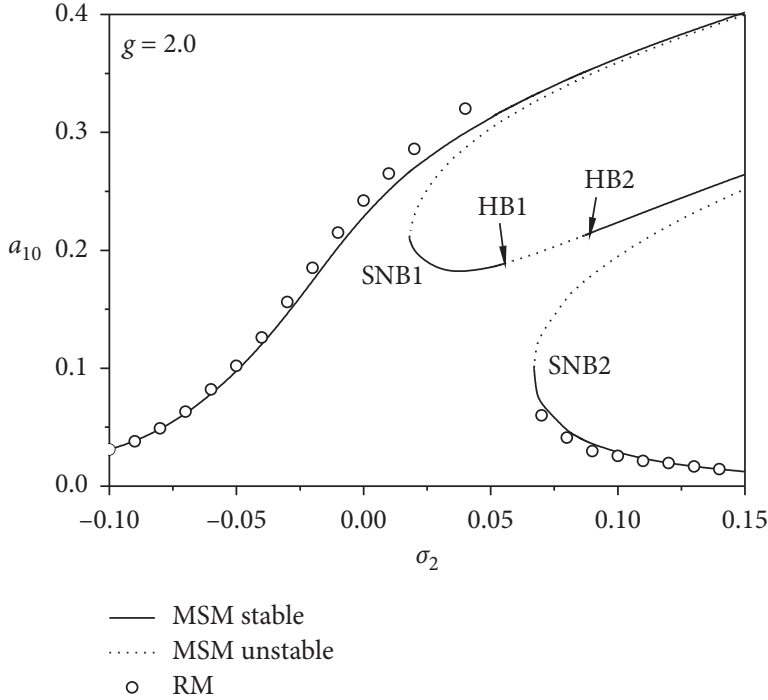

(a)

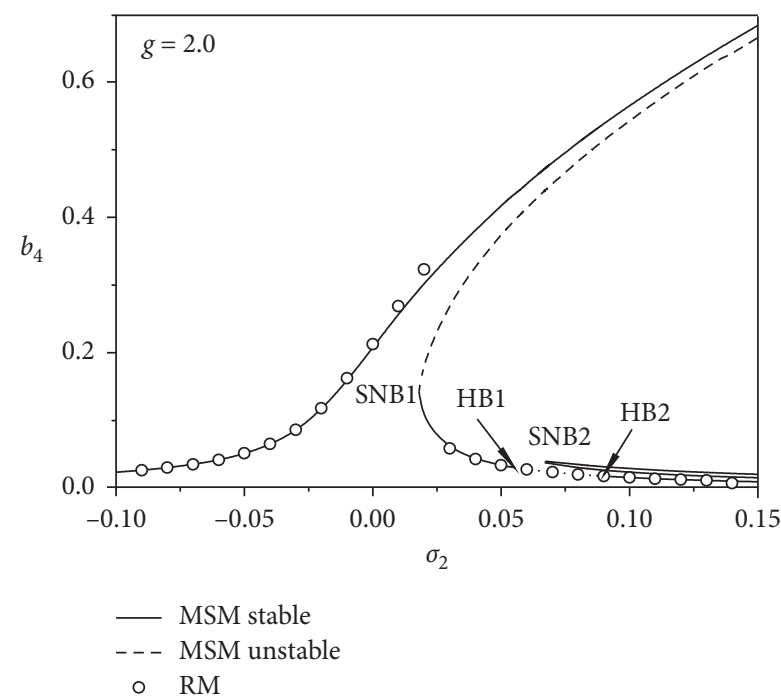

(b)

FIgURE 7: Frequency-response curves of the cable and shallow arch with $g_{4}=2.0$ and $\bar{\Omega}_{4} \approx \omega_{2,4}$.

stimulated has a larger amplitude. As described in Figure 7, in this case, the cable vibration of cable dominates the nonlinear response. On the contrary, in order to verify the approximate analytical solutions obtained with multiple scale method (MSM), Runge-Kutta method (RM) is applied to the original ODEs in equations (8) and (9), and the corresponding numerical solutions are also presented in Figure 7. As shown in Figure 7, the solid lines are stable solutions obtained from MSM, dashed lines are unstable solutions, and circles are the solution obtained from RM. According to the error analysis, the present results have a good agreement with the numerical results obtained from RM (Table 3).

Figure 8 shows the force-response curves with various excitation amplitudes and detuning parameters. When the detuning parameter $\sigma_{2}<0$, there is a single-valued relationship between the response and excitation amplitudes. When $\sigma_{2} \geq 0$, there is a relatively small range of multiple solutions of the system because of the saddle-node bifurcations at SNB1 and SNB2. There are two stable solutions and an unstable solution between SNB1 and SNB2. When $g$ increases from SNB1 to SNB2, the two stable equilibrium solutions coexist.

Figure 9 shows the time histories of the cable and shallow arch, obtained by integration of two degrees-of-freedom ordinary differential equations in equations (8) and (9) under the initial conditions where the initial speed and displacement are zeros.

3.2. Dynamic Solutions, Bifurcation, and Chaos. The Cartesian form of the equations has the following standard form:

$$
\dot{x}=F(x) \text {. }
$$

The periodic solutions and chaotic solutions can be obtained by the shooting method. After the periodic solution
TABLE 3: Analytical solutions and numerical solutions of the cablestayed shallow arch.

\begin{tabular}{lcccccc}
\hline \multirow{2}{*}{$\sigma_{2}$} & \multicolumn{2}{c}{ MSM } & \multicolumn{2}{c}{ RM } & \multicolumn{2}{c}{ Difference ratio } \\
& $a_{10}$ & $b_{4}$ & $a_{10}$ & $b_{4}$ & $a_{10}(\%)$ & $b_{4}(\%)$ \\
\hline-0.09 & 0.038 & 0.0264 & 0.038 & 0.0264 & 0.0 & 0.0 \\
-0.08 & 0.048 & 0.0301 & 0.049 & 0.0303 & 2.1 & 0.7 \\
-0.07 & 0.061 & 0.0349 & 0.063 & 0.0351 & 3.3 & 0.6 \\
-0.06 & 0.078 & 0.0414 & 0.082 & 0.0418 & 5.1 & 1.0 \\
-0.05 & 0.097 & 0.0506 & 0.102 & 0.0515 & 5.2 & 1.8 \\
-0.04 & 0.120 & 0.0640 & 0.126 & 0.0652 & 5.0 & 1.9 \\
-0.03 & 0.146 & 0.0843 & 0.156 & 0.0861 & 6.8 & 2.1 \\
-0.02 & 0.174 & 0.1154 & 0.185 & 0.1179 & 6.3 & 2.2 \\
-0.01 & 0.203 & 0.1585 & 0.215 & 0.1622 & 5.9 & 2.3 \\
0 & 0.229 & 0.2080 & 0.242 & 0.2128 & 5.7 & 2.3 \\
\hline
\end{tabular}

$x_{0}$ is constructed, the branch of periodic solution is tracked by pseudoarclength path-following algorithm, and its stability is determined using the Floquet theory.

Figure 10 shows the phase portraits and Poincare maps of the dynamic solutions onto the $u_{10}-v_{10}$ plane as the detuning parameter $\sigma_{2}$ slowly varies. As $\sigma_{2}$ increases from 0.05 , a one-period limit cycle develops, as shown in Figure 10(a) $\left(\sigma_{2}=0.05587\right)$. The stable 1P solution then loses its stability via a period-doubling bifurcation, and a twoperiod solution is shown in Figure 10(b) $\left(\sigma_{2}=0.05602\right)$. As $\sigma_{2}$ increases further, the $2 \mathrm{P}$ solution undergoes a cascade of period-doubling bifurcations at $\sigma_{2}=0.05635$ (4P). Finally, this cascade of period-doubling bifurcations leads to chaos. A representative chaotic attractor is shown in Figure 10(d).

Figure 11 shows the phase portraits and Poincare maps of the dynamic solutions onto the $u_{4}-v_{4}$ plane as the detuning parameter $\sigma_{2}$ slowly varies. When $\sigma_{2}$ increases past Hopf bifurcation point (HB1), a small limit cycle develops and grows in size, deforms, and then undergoes a sequence of period-doubling bifurcations leading to chaos, as shown in Figures 11(a)-11(d). 


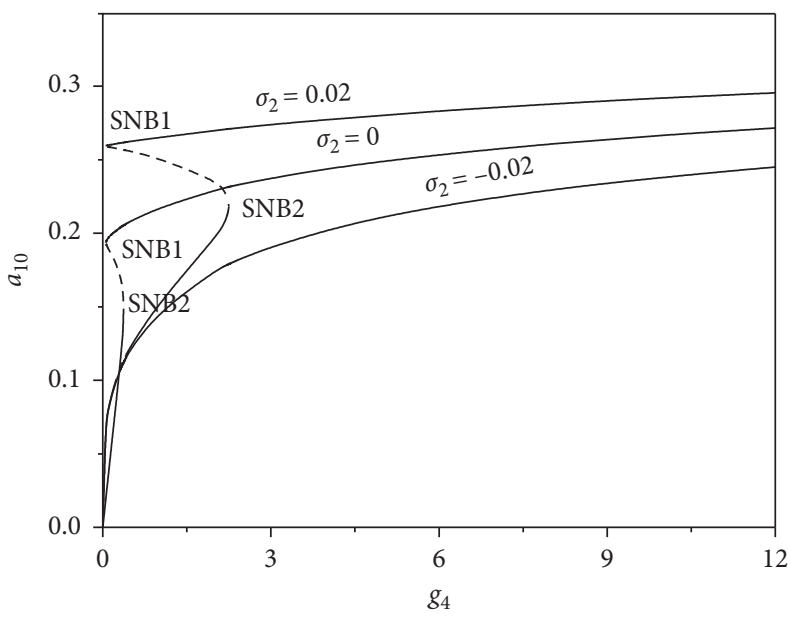

(a)

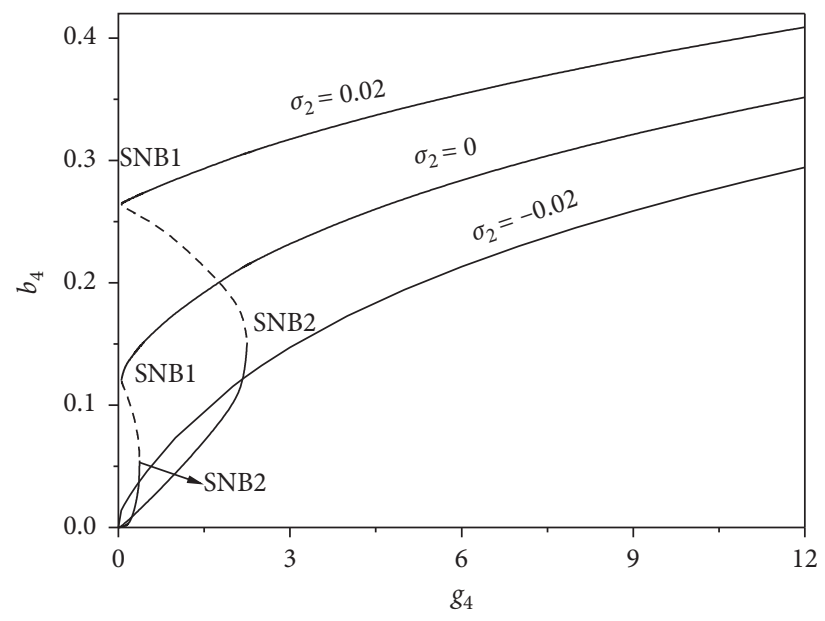

(b)

Figure 8: Variations in the force-response curves of the (a) cable and (b) arch with $g$ and $\sigma_{2}$ when $\bar{\Omega}_{4} \approx \omega_{2,4}$.

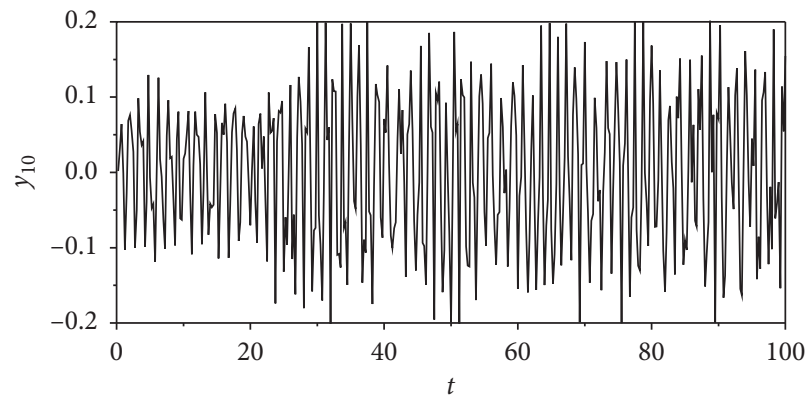

(a)

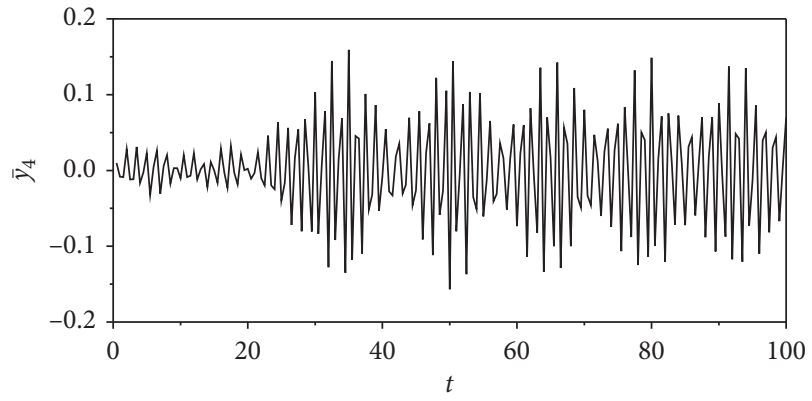

(b)

Figure 9: Time histories of cable (a) and shallow arch (b) with $\sigma_{2}=0.056$ and $g_{4}=2.0$.

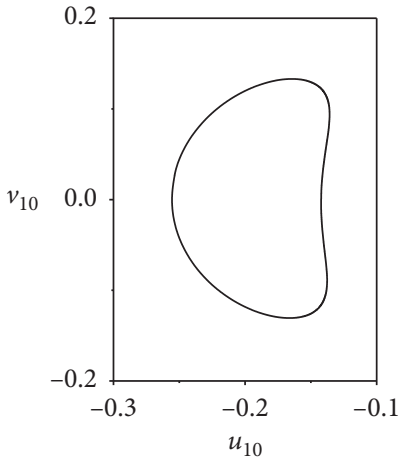

(a)

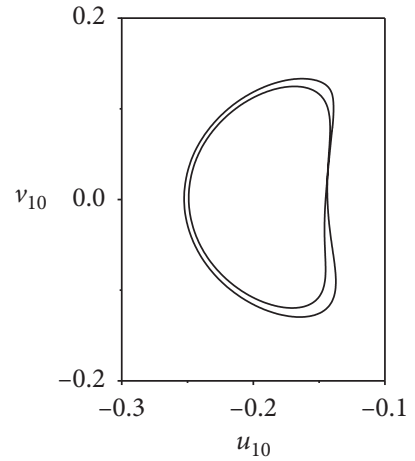

(b)

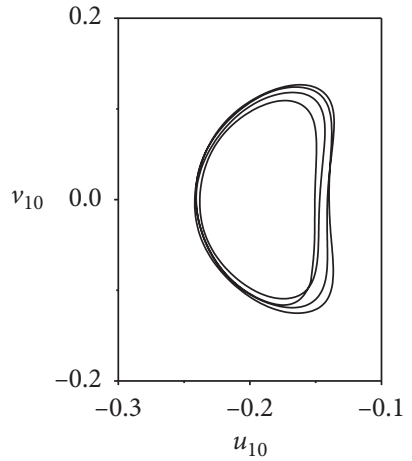

(c)

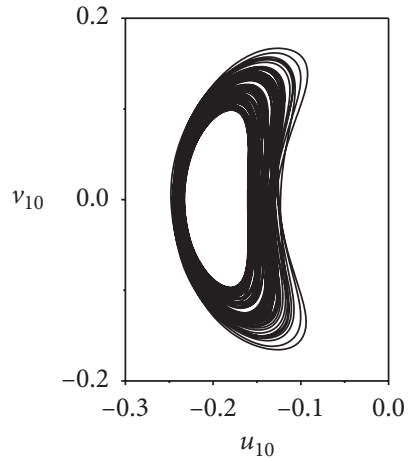

(d)

Figure 10: Continued. 


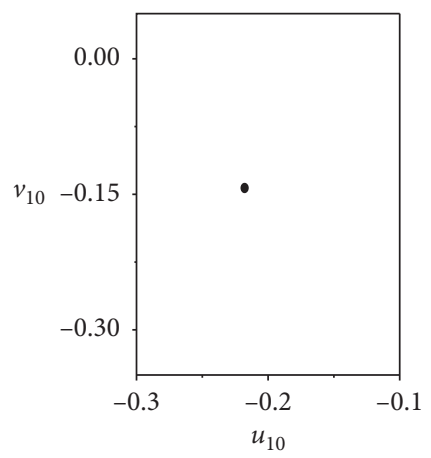

(e)

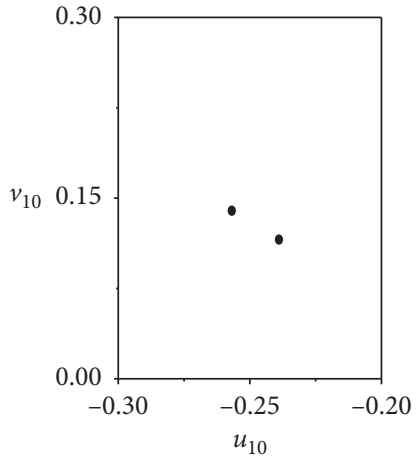

(f)

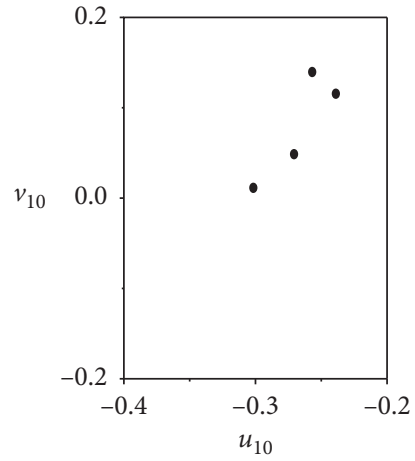

(g)

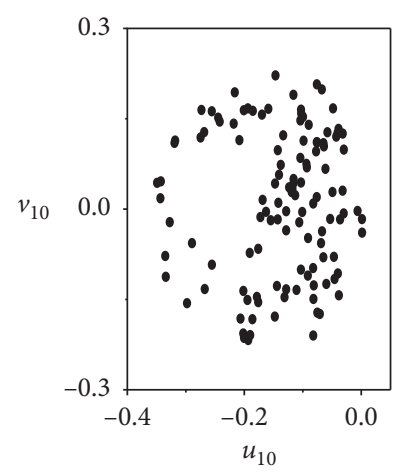

(h)

FIgURE 10: Phase portraits and Poincare maps of the dynamic solutions onto the $u_{10}-v_{10}$ phase. (a, e) $\sigma_{2}=0.05587(1 \mathrm{P}) ;(\mathrm{b}, \mathrm{f}) \sigma_{2}=0.05602$ $(2 \mathrm{P}) ;(\mathrm{c}, \mathrm{g}) \sigma_{2}=0.05634(4 \mathrm{P}) ;(\mathrm{d}, \mathrm{h}) \sigma_{2}=0.05658$ (chaos).

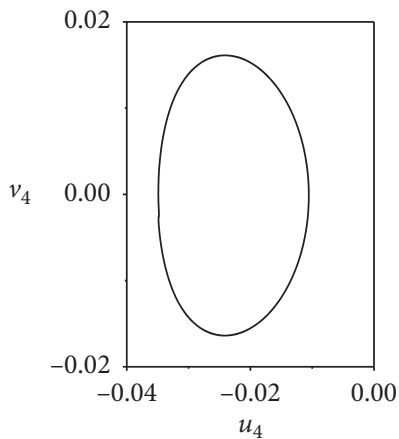

(a)

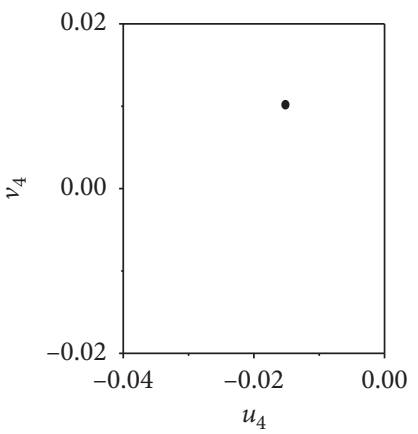

(e)

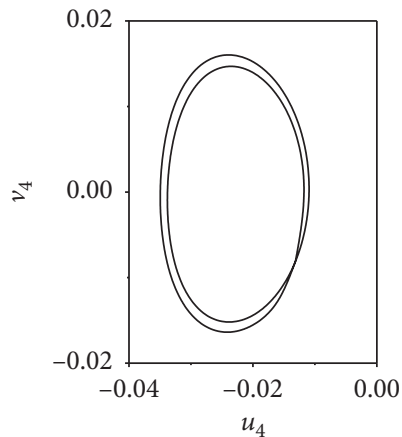

(b)

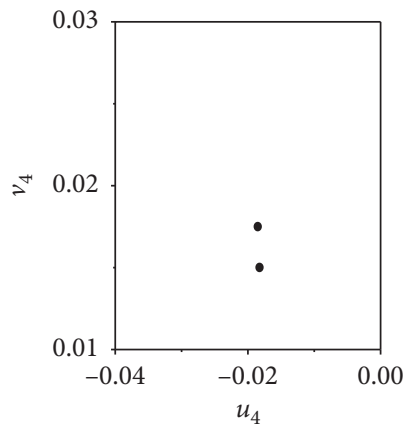

(f)

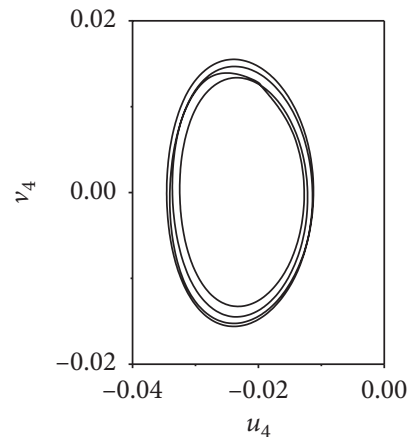

(c)

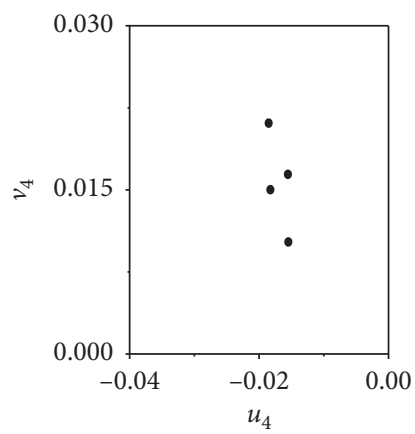

(g)

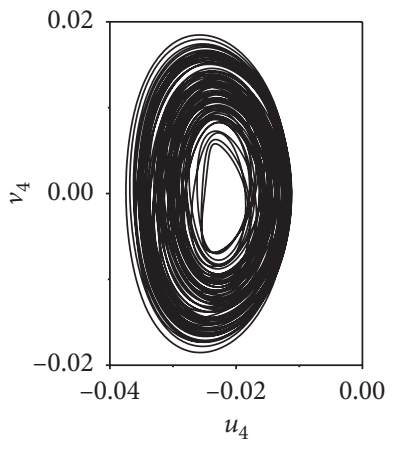

(d)

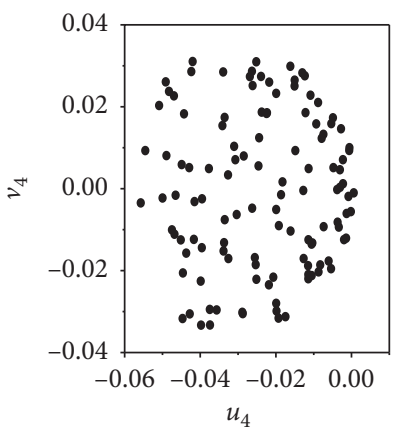

(h)

Figure 11: Phase portraits and Poincare maps of the dynamic solutions onto the $u_{4}-v_{4}$ phase. (a, e) $\sigma_{2}=0.05587(1 \mathrm{P}) ;(\mathrm{b}, \mathrm{f}) \sigma_{2}=0.05602$ $(2 \mathrm{P}) ;(\mathrm{c}, \mathrm{g}) \sigma_{2}=0.05634(4 \mathrm{P}) ;(\mathrm{d}, \mathrm{h}) \sigma_{2}=0.05658$ (chaos).

Figure 12 shows the bifurcation diagrams as the detuning parameter $\sigma_{2}$ is varied in the range $0.0556 \leq \sigma_{2} \leq 0.0568$. The values of other parameters are all fixed when there is $1: 1$ internal resonance and simultaneous primary resonance. As seen in Figure 12, as the detuning parameter $\sigma_{2}$ slowly increases in the narrow region $\sigma_{2} \in(0.0556,0.0568)$, and motion of the system undergoes the following process: chaotic motion $\longrightarrow$ periodic-1 motion $\longrightarrow$ periodic-2 motion $\longrightarrow \cdots \longrightarrow$ chaotic motion. In addition, it should be mentioned that the chaos region on the left of Figure 12 belonged to another bifurcation interval.
3.3. Effects of Key Parameters. In this section, the effects of the key parameters of the cable and shallow arch on the frequency-response curves of the cable-stayed shallow arch are investigated. The frequency-response curves shown in Figures 13-16 are obtained by numerical calculation with different values of parameters. These key parameters include the initial tension, the inclined angle of the cable, and the rise and inclined angle of shallow arch.

In order to investigate the effect of the initial tension $\mathrm{H}$ of the cable on the dynamic behavior of the system, Figure 13 illustrates the frequency-response curves under different 


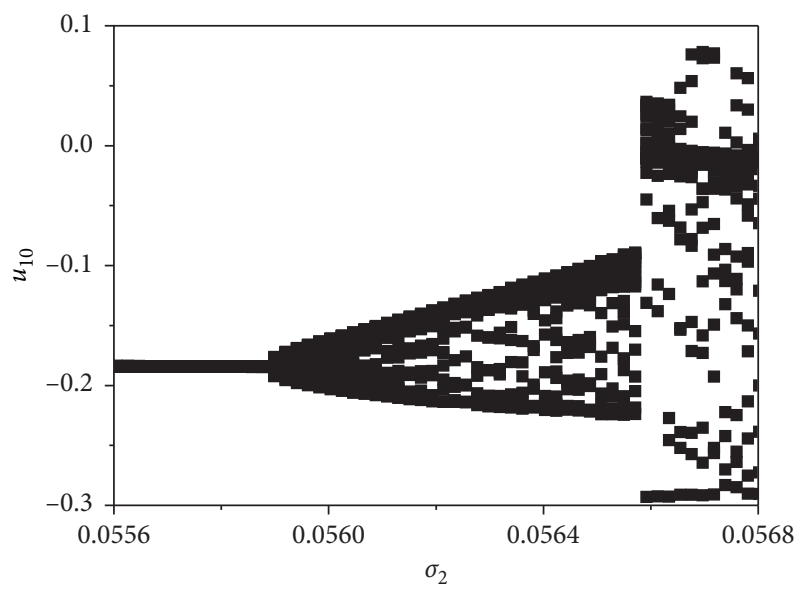

(a)

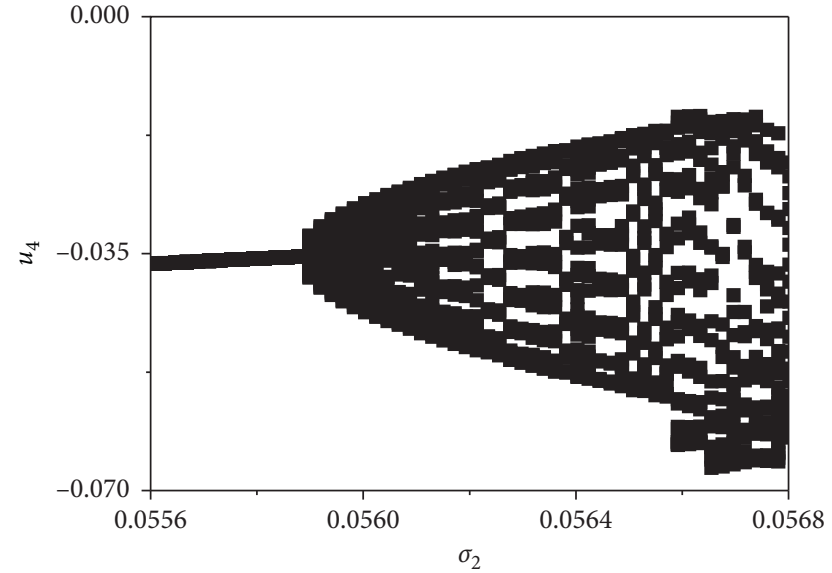

(b)

Figure 12: Bifurcation diagrams of the cable (a) and shallow arch (b) with the excitation acting on the arch.

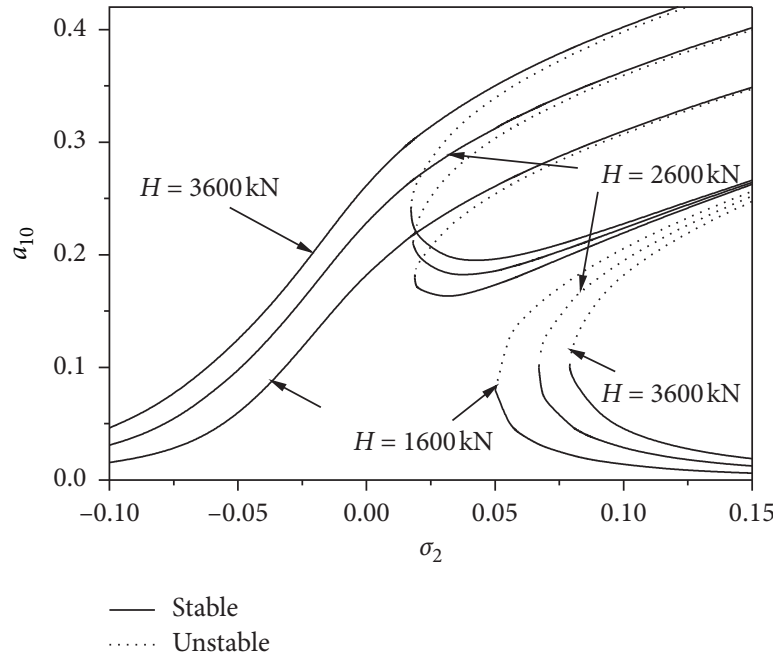

(a)

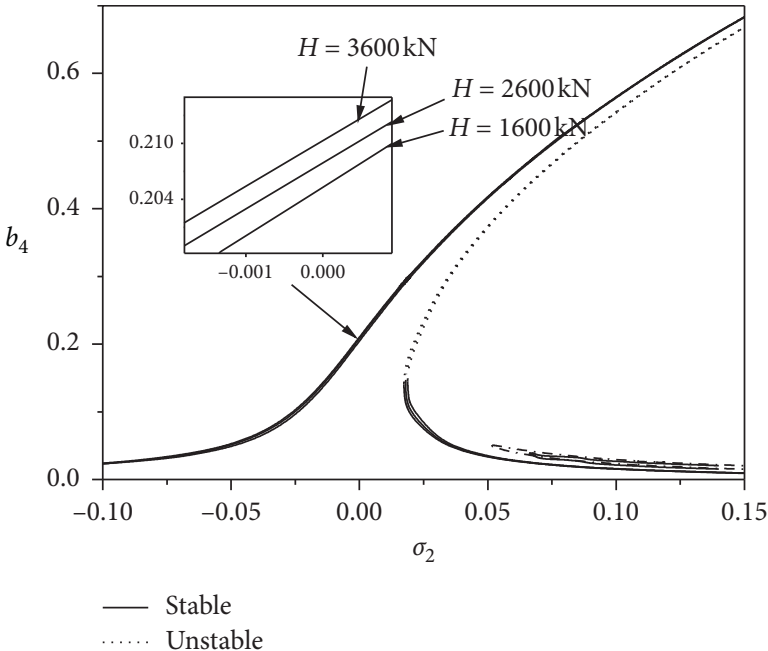

(b)

FIGURE 13: Effect of initial tension $(H)$ on the frequency-response curves of the (a) cable and (b) shallow arch with $\bar{\Omega}_{4} \approx \omega_{2,4}$ and $\omega_{1,10} \approx \omega_{2,4}$.

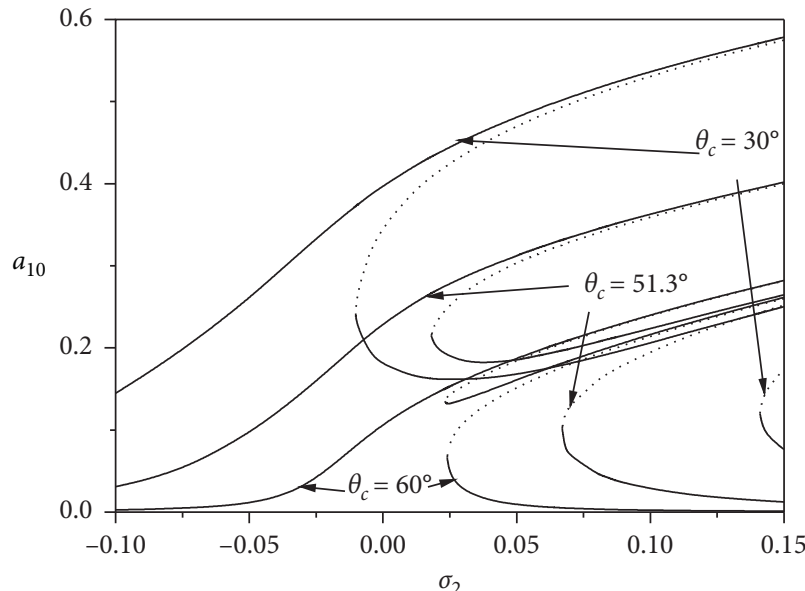

- Stable Unstable

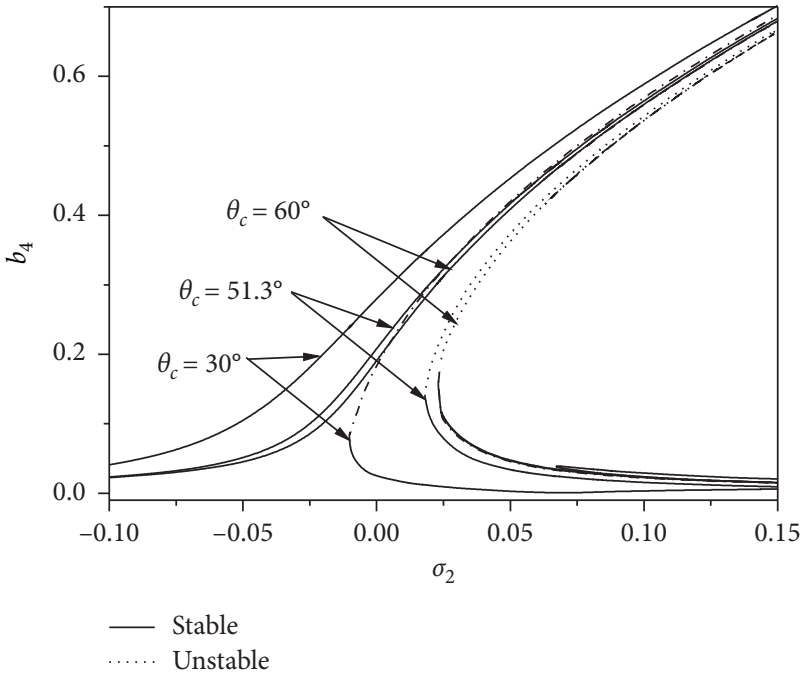

(b)

FIGURE 14: Effect of inclined angle $\theta_{c}$ on the frequency-response curves of the (a) cable and (b) shallow arch with $\bar{\Omega}_{4} \approx \omega_{2,4}$ and $\omega_{1,10} \approx \omega_{2,4}$. 


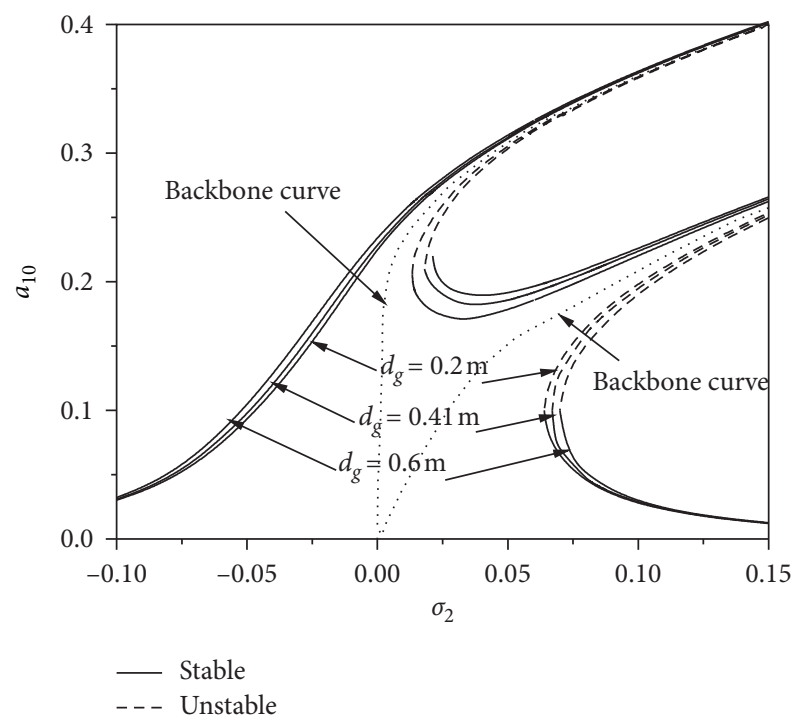

(a)

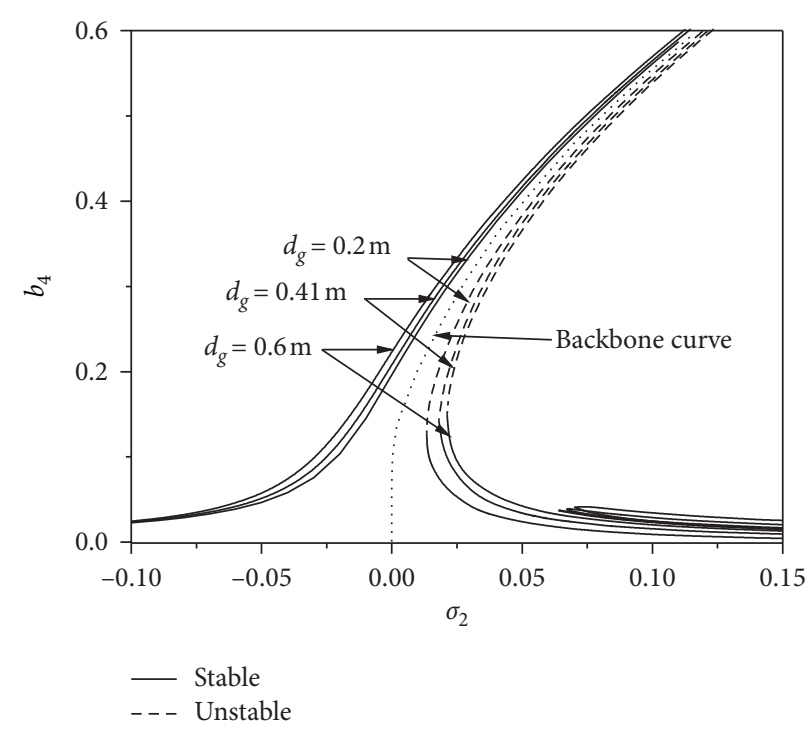

(b)

Figure 15: Effect of the arch rise $d_{g}$ on the frequency-response curves of the (a) cable and (b) shallow arch with $\bar{\Omega}_{4} \approx \omega_{2,4}$ and $\omega_{1,10} \approx \omega_{2,4}$.

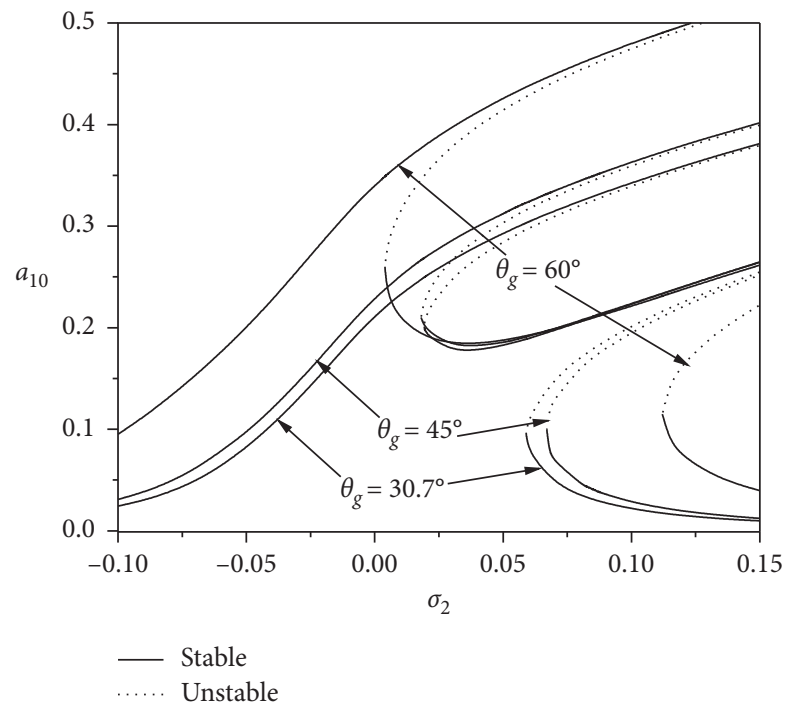

(a)

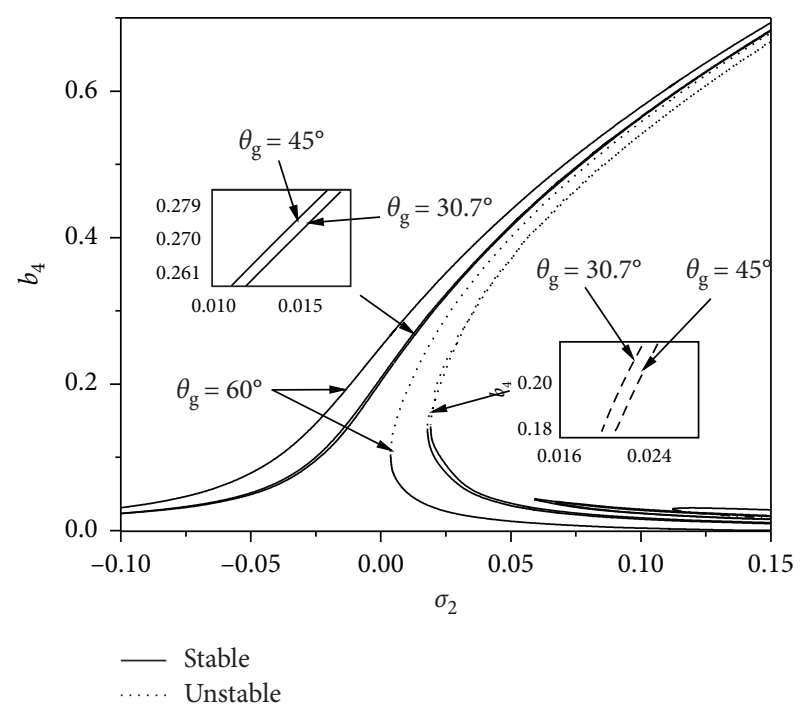

(b)

FIGURE 16: Effect of arch elevation $\theta_{g}$ on the frequency-response curves of the (a) cable and (b) shallow arch with $\bar{\Omega}_{4} \approx \omega_{2,4}$ and $\omega_{1,10} \approx \omega_{2,4}$.

values of detuning parameter $\sigma_{2}$ when $\bar{\Omega}_{4} \approx \omega_{2,4}$ and $\omega_{1,10} \approx \omega_{2,4}$. As shown in Figure 13, the initial tension governs the multivalue region of the frequency-response curves. The resonance interval of the system is increased when the initial tension of the cable increases. With the increase in initial tension, the response amplitudes of the system also increase. Overall, the initial tension has a significant effect on the frequency-response curves of the cable, as shown in Figure 13(a), whereas the influence of parameter on the frequency-response curves of the shallow arch is not susceptible as seen in Figure 13(b).
Generally, the inclined angle $\theta_{c}$ of the cable plays an important role in the dynamic behavior of the cablestayed shallow arch. Figure 14 shows the effect of the inclined angle of the cable on the frequency-response curves of the cable-stayed system when $\bar{\Omega}_{4} \approx \omega_{2,4}$ and $\omega_{1,10} \approx \omega_{2,4}$. As expected, increasing or decreasing the inclined angle causes significant changes in the frequencyresponse curves. As can be seen in Figure 14, as the inclined angle is increased, the response amplitudes of the system decrease and the hardening spring behavior of the resonance increases. Additionally, increasing the inclined angle delays the jump phenomenon of the frequency- 
response curves. It should be noted that the three-valued region of the cable rapidly narrows and the five-valued region gets larger. That is, the resonance interval of the system decreases with the increase in the inclined angle. However, the inclined angle parameter has no obvious impact on the multivalue range of the shallow arch.

Figure 15 shows the effect of the shallow arch rise $d_{g}$ on the dynamic behavior of the cable-stayed shallow arch with $\bar{\Omega}_{4} \approx \omega_{2,4}$ and $\omega_{1,10} \approx \omega_{2,4}$. As shown in Figure 15, a backbone curve exists for the three different frequencyresponse curves with each different arch rise. The fivevalued region of the cable slow decreases as the arch rise value is decreased, which is in a similar trend to that of the initial tension on the dynamic response. However, in contrast with the results of the initial tension on the dynamic behavior, the arch rise has a slight effect on the response amplitude of the resonance.

In the cable-stayed shallow arch structure, the shallow arch inclined angle $\theta_{g}$ is another key parameter of the structure. Figure 16 shows the effect of shallow arch inclined angle on the dynamic behavior of the system when $\bar{\Omega}_{4} \approx \omega_{2,4}$ and $\omega_{1,10} \approx \omega_{2,4}$. As shown in Figure 16, the arch elevation has a significant influence on the multivalue region of the cable, and the jump phenomenon of the shallow arch can be delayed by decreasing the shallow arch inclined angle. In particular, the multivalue range of the cable rapidly expands for shallow arch inclined angle above $45^{\circ}$. The shallow arch inclined angle has little effect on the multivalue region of the shallow arch.

\section{Conclusions}

The 1:1 internal resonance characteristics of cable-stayed shallow have been investigated in this paper. Analytical solution for $1: 1$ internal resonance and primary resonance has been derived for the cable-stayed shallow arch. The effects of initial tension, inclined angle, arch rise, and elevation on the internal resonance responses have been discussed in detail. It is found that the $1: 1$ internal resonance of cable-stayed shallow arch may occur when the natural frequency of local mode for the cable is close to that of global mode for the shallow arch. Numerical results also show that periodic motion of the system may lose its stability due to the periodic-doubling bifurcation. It is also found that the effects of the inclined angle of both cable and shallow arch on the dynamic characteristics of the cable-stayed shallow arch system are quite remarkable, while the effects of the rise of shallow arch and the initial tension of the cable are not. The comprehensive numerical results and research findings will provide essential information for the safety evaluation of cable-supported structures that are widely used in civil engineering.

\section{Appendix}

$$
\begin{aligned}
& \omega_{1, n}^{2}=\frac{a_{3}^{n i}}{a_{1}^{n i}}, \\
& \omega_{2, n}^{2}=\frac{b_{3}^{n i}}{b_{1}^{n i}}, \\
& 2 \mu_{1, n}=\frac{a_{2}^{n i}}{a_{1}^{n i}}, \\
& 2 \mu_{2, n}=\frac{b_{2}^{n i}}{b_{1}^{n i}}, \\
& \alpha_{1, m n}=\frac{a_{3}^{m i}}{a_{1}^{n i}}(m \neq n), \\
& \alpha_{2, m n}=\frac{b_{3}^{m i}}{b_{1}^{n i}}(m \neq n) \text {, } \\
& \alpha_{3, m n}=\frac{a_{4}^{m i}}{a_{1}^{n i}}, \\
& \alpha_{4, m n}=\frac{b_{4}^{m i}}{b_{1}^{n i}}, \\
& \beta_{1, m p n}=\frac{a_{5}^{m p i}}{a_{1}^{n i}}, \\
& \beta_{2, m p n}=\frac{b_{5}^{m p i}}{b_{1}^{n i}}, \\
& \beta_{3, m p n}=\frac{a_{6}^{m p i}}{a_{1}^{n i}}, \\
& \beta_{4, m p n}=\frac{b_{6}^{m p i}}{b_{1}^{n i}}, \\
& \gamma_{1, m p q n}=\frac{a_{7}^{m p q i}}{a_{1}^{n i}}, \\
& \gamma_{2, m p q n}=\frac{b_{7}^{m p q i}}{b_{1}^{n i}}, \\
& g_{n}=\frac{G_{n}}{b_{1}^{n i}}
\end{aligned}
$$


where

$$
\begin{aligned}
& a_{1}^{n i}=\int_{0}^{l_{c}} m_{c} \varphi_{n}(x) \varphi_{i}(x) \mathrm{d} x, \\
& a_{2}^{n i}=\int_{0}^{l_{c}} c_{c} \varphi_{n}(x) \varphi_{i}(x) \mathrm{d} x, \\
& a_{3}^{m i}=\int_{0}^{l_{c}} E_{c} A_{c} y_{0}^{\prime \prime} \frac{\varphi_{m}\left(l_{c}\right)}{l_{c} \tan \left(\theta_{g}+\theta_{c}\right)} \varphi_{i}(x) \mathrm{d} x-\int_{0}^{l_{c}} H \varphi_{i}(x) \varphi_{m}^{\prime \prime}(x) \mathrm{d} x-\int_{0}^{l_{c}} E_{c} A_{c} y_{0}^{\prime \prime} \frac{1}{l_{c}} \int_{0}^{l_{c}} y_{0}^{\prime} \varphi_{m}^{\prime}(x) \mathrm{d} x \varphi_{i}(x) \mathrm{d} x, \\
& a_{4}^{m i}=-\int_{0}^{l_{c}} E_{c} A_{c} y_{0}^{\prime \prime} \frac{\phi_{m}\left(l_{g}\right)}{l_{c} \sin \left(\theta_{g}+\theta_{c}\right)} \varphi_{i}(x) \mathrm{d} x, \\
& a_{5}^{m p i}=-\int_{0}^{l_{c}} E_{c} A_{c} \varphi_{m}^{\prime \prime}(x) \frac{\phi_{p}\left(l_{g}\right)}{l_{c} \sin \left(\theta_{g}+\theta_{c}\right)} \varphi_{i}(x) \mathrm{d} x, \\
& a_{6}^{m p i}=\int_{0}^{l_{c}} E_{c} A_{c} \varphi_{m}^{\prime \prime}(x) \frac{\varphi_{p}\left(l_{c}\right)}{l_{c} \sin \left(\theta_{g}+\theta_{c}\right)} \varphi_{i}(x) \mathrm{d} x-\int_{0}^{l_{c}} E_{c} A_{c} y_{0}^{\prime \prime} \frac{1}{2 l_{c}} \int_{0}^{l_{c}} \varphi_{m}^{\prime}(x) \varphi_{p}^{\prime}(x) \mathrm{d} x \varphi_{i}(x) \mathrm{d} x \\
& -\int_{0}^{l_{c}} E_{c} A_{c} \varphi_{m}^{\prime \prime}(x) \frac{1}{l_{c}} \int_{0}^{l_{c}} y_{0}^{\prime} \varphi_{p}^{\prime}(x) \mathrm{d} x \varphi_{i}(x) \mathrm{d} x, \\
& a_{7}^{m p q i}=-\int_{0}^{l_{c}} E_{c} A_{c} \varphi_{m}^{\prime \prime}(x) \frac{1}{2 l_{c}} \int_{0}^{l_{c}} \varphi_{p}^{\prime}(x) \varphi_{q}^{\prime}(x) \mathrm{d} x \varphi_{i}(x) \mathrm{d} x, \\
& b_{1}^{n i}=\int_{0}^{l_{g}} m_{g} \phi_{i}(\bar{x}) \phi_{n}(\bar{x}) d \bar{x}, \\
& b_{2}^{n i}=\int_{0}^{l_{g}} c_{g} \phi_{i}(\bar{x}) \phi_{n}(\bar{x}) d \bar{x} \\
& b_{3}^{m i}=\int_{0}^{l_{g}} E_{g} I_{g} \phi_{n}^{(4)}(\bar{x}) \phi_{i}(\bar{x}) \mathrm{d} \bar{x}-\int_{0}^{l_{g}} N \phi_{n}^{\prime \prime}(\bar{x}) \phi_{i}(\bar{x}) \mathrm{d} \bar{x}+\int_{0}^{l_{g}} E_{g} A_{g}^{-} y_{0}^{\prime \prime}\left(\frac{\phi_{p}\left(l_{g}\right)}{l_{g} \tan \left(\theta_{g}+\theta_{c}\right)}\right) \phi_{i}(\bar{x}) \mathrm{d} \bar{x} \\
& -\int_{0}^{l_{g}} E_{g} A_{g}^{-} y_{0}^{\prime \prime} \frac{1}{l_{g}} \int_{0}^{l_{g}} y_{0}^{\prime} \phi_{p}^{\prime}(\bar{x}) \mathrm{d} \bar{x} \phi_{i}(\bar{x}) \mathrm{d} \bar{x}, \\
& b_{4}^{m i}=-\int_{0}^{l_{g}} E_{g} A_{g}^{-} y_{0}^{\prime \prime} \frac{\varphi_{m}\left(l_{c}\right)}{l_{g} \sin \left(\theta_{g}+\theta_{c}\right)} \phi_{i}(\bar{x}) \mathrm{d} \bar{x}, \\
& b_{5}^{m p i}=-\int_{0}^{l_{g}} E_{g} A_{g} \phi_{m}^{\prime \prime}(\bar{x}) \frac{\varphi_{p}\left(l_{c}\right)}{l_{g} \sin \left(\theta_{g}+\theta_{c}\right)} \phi_{i}(\bar{x}) \mathrm{d} \bar{x}, \\
& b_{6}^{m p i}=\int_{0}^{l_{g}} E_{g} A_{g} \phi_{m}^{\prime \prime}(\bar{x})\left(\frac{\phi_{p}\left(l_{g}\right)}{l_{g} \tan \left(\theta_{g}+\theta_{c}\right)}\right) \phi_{i}(\bar{x}) \mathrm{d} \bar{x}-\int_{0}^{l_{g}} E_{g} A_{g}^{-} y_{0}^{\prime \prime} \frac{1}{2 l_{g}} \int_{0}^{l_{g}} \phi_{m}^{\prime}(\bar{x}) \phi_{p}^{\prime}(\bar{x}) \mathrm{d} \bar{x} \phi_{i}(\bar{x}) \mathrm{d} \bar{x} \\
& -\int_{0}^{l_{g}} E_{g} A_{g} \phi_{m}^{\prime \prime}(\bar{x}) \frac{1}{l_{g}} \int_{0}^{l_{g}} y_{0}^{\prime} \phi_{p}^{\prime}(\bar{x}) \mathrm{d} \bar{x} \phi_{i}(\bar{x}) \mathrm{d} \bar{x} \\
& b_{7}^{m p q i}=-\int_{0}^{l_{g}} E_{g} A_{g} \phi_{m}^{\prime \prime}(\bar{x}) \frac{1}{2 l_{g}} \int_{0}^{l_{g}} y_{0}^{\prime}(\bar{x}) \phi_{q}^{\prime}(\bar{x}) \mathrm{d} \bar{x} \phi_{i}(\bar{x}) \mathrm{d} \bar{x}, \\
& G_{n}=\int_{0}^{l_{g}} G(\bar{x}) \phi_{i}(\bar{x}) \mathrm{d} \bar{x} .
\end{aligned}
$$




\section{Data Availability}

The data used to support the findings of this study are included within the article.

\section{Conflicts of Interest}

The authors declare that there are no conflicts of interest regarding the publication of this article.

\section{Acknowledgments}

The study was supported by the National Natural Science Foundation of China (Grant no. 51678247).

\section{References}

[1] R. Barsotti, S. S. Ligaro, and G. F. Royer-Carfagni, "The web bridge," International Journal of Solids and Structures, vol. 38, no. 48-49, pp. 8831-8850, 2001.

[2] J. H. G. Macdonald, "Separation of the contributions of aerodynamic and structural damping in vibrations of inclined cables," Journal of Wind Engineering and Industrial Aerodynamics, vol. 90, no. 1, pp. 19-39, 2002.

[3] A. M. Abdel-Ghaffar and M.,A. Khalifa, "Importance of cable vibration in dynamics of cable-stayed beam," ASCE Journal of Engineering Mechanics, vol. 117, no. 11, pp. 2571-2589, 1991.

[4] J. F. Fleming and E. A. Egeseli, "Dynamic behaviour of a cablestayed bridge," Earthquake Engineering \& Structural Dynamics, vol. 8, no. 1, pp. 1-16, 1980.

[5] F. Benedettini, G. Rega, and R. Alaggio, "Non-linear oscillations of a four-degree-of-freedom model of a suspended cable under multiple internal resonance conditions," Journal of Sound and Vibration, vol. 182, no. 5, pp. 775-798, 1995.

[6] T. D. Guo, H. J. Kang, L. H. Wang, and Y. Y. Zhao, "Cable's mode interactions under vertical support motions:boundary resonant modulation," Nonlinear Dynamics, vol. 83, pp. 1577-1600, 2016.

[7] S. Y. Lee, J. J. Sheu, and S. M. Lin, "In-plane vibrational analysis of rotating curved beam with elastically restrained root," Journal of Sound and Vibration, vol. 315, no. 4-5, pp. 1086-1102, 2008.

[8] Z. P. Yi and S. Ilinca, "Nonlinear normal modes of a shallow arch with elastic constraints for two-to-one internal resonances," Nonlinear Dyn, vol. 84, pp. 1259-1279, 2016.

[9] A. S. Nazmy and A. M. Abdel-Ghaffar, "Non-linear earthquake-response analysis of long-span cable-stayed bridges: Theory," Earthquake Engineering \& Structural Dynamics, vol. 19 , no. 1 , pp. 45-62, 1990.

[10] A. S. Nazmy and A. M. Abdel-Ghaffar, "Non-linear earthquake-response analysis of long-span cable-stayed bridges: Applications," Earthquake Engineering \& Structural Dynamics, vol. 19, no. 1, pp. 63-76, 1990.

[11] Y. Fujino, P. Warnitchai, and B. M. Pacheco, "An experimental and analytical study of autoparametric resonance in a 3DOF model of cable-stayed-beam," Nonlinear Dynamics, vol. 4, no. 2, pp. 111-138, 1993.

[12] P. Warnitchai, Y. Fujino, B. M. Pacheco, and R. Agret, "An experimental study on active tendon control of cable-stayed bridges," Earthquake Engineering \& Structural Dynamics, vol. 22, no. 2, pp. 93-111, 1993.

[13] P. Warnitchai, Y. Fujino, and T. Susumpow, "A non-linear dynamic model for cables and its application to a cable-structure system," Journal of Sound and Vibration, vol. 187, no. 4, pp. 695-712, 1995.

[14] V. Gattulli, M. Morandini, and A. Paolone, "A parametric analytical model for non-linear dynamics in cable-stayed beam," Earthquake Engineering \& Structural Dynamics, vol. 31, no. 6, pp. 1281-1300, 2002.

[15] V. Gattulli and M. Lepidi, "Nonlinear interactions in the planar dynamics of cable-stayed beam," International Journal of Solids and Structures, vol. 40, no. 18, pp. 4729-4748, 2003.

[16] V. Gattulli, M. Lepidi, J. H. G. Macdonald, and C. A. Taylor, "One-to-two global-local interaction in a cable-stayed beam observed through analytical, finite element and experimental models," International Journal of Non-linear Mechanics, vol. 40, no. 4, pp. 571-588, 2005.

[17] V. Gattulli and M. Lepid, "Localization and veering in the dynamics of cable-stayed bridges," Computers \& Structures, vol. 85, no. 21-22, pp. 1661-1678, 2007.

[18] M. H. Wei, Y. Q. Xiao, and H. T. Liu, "Bifurcation and chaos of a cable-beam coupled system under simultaneous internal and external resonances," Nonlinear Dynamics, vol. 67, no. 3, pp. 1969-1984, 2012.

[19] H. J. Kang, H. P. Zhu, Y. Y. Zhao, and Z. P. Yi, "In-plane nonlinear dynamics of the stay cables," Nonlinear Dynamics, vol. 73, no. 3, pp. 1385-1398, 2013.

[20] Z. Wang, C. Sun, Y. Zhao, and Z. Yi, "Modeling and nonlinear modal characteristics of the cable-stayed beam," European Journal of Mechanics-A/Solids, vol. 47, pp. 58-69, 2014.

[21] N. Srinil and G. Rega, "Space-time numerical simulation and validation of analytical predictions for nonlinear forced dynamics of suspended cables," Journal of Sound and Vibration, vol. 315, no. 3, pp. 394-413, 2008.

[22] A. Luongo and D. Zulli, "Nonlinear energy sink to control elastic strings: the internal resonance case," Nonlinear Dynamics, vol. 81, no. 1-2, pp. 425-435, 2015.

[23] J. S. Ju and Y. L. Guo, "In-plane elastic buckling of arch," Tsinghua Science and Technology, vol. 7, no. 3, pp. 322-325, 2002.

[24] Y. M. Ai, B. C. Yang, and P. M. Huang, "Practical calculation of critical lateral flexure load of cable-arch systems," Journal of Jilin University (Engineering and Technology Edition), vol. 40, no. 1, pp. 63-66, 2010.

[25] Y. Zhao and H. Kang, "In-plane free vibration analysis of cable-arch structure," Journal of Sound and Vibration, vol. 312, no. 3, pp. 363-379, 2008.

[26] H. J. Kang, Y. Y. Zhao, and H. P. Zhu, "Out-of-plane free vibration analysis of a cable-arch structure," Journal of Sound and Vibration, vol. 332, no. 4, pp. 907-921, 2013.

[27] J. G. Lv, Y. Y. Zhao, and R. H. Wang, "Dynamical modeling and internal resonance of cable-stayed arch structure," Journal of Central South University (Science and Technology), vol. 41, no. 1, pp. 316-321, 2010.

[28] H. J. Kang, T. D. Guo, Y. Y. Zhao, W. B. Fu, and L. H. Wang, "Dynamic modeling and in-plane 1:1:1 internal resonance analysis of cable-stayed bridge," European Journal of Mechanics-A/Solids, vol. 62, pp. 94-109, 2017.

[29] F. Lakrad and W. Schiehlen, "Effects of a low frequency parametric excitation," Chaos, Solitons \& Fractals, vol. 22, no. 5, pp. 1149-1164, 2004.

[30] W. Lacarbonara, G. Rega, and A. H. Nayfeh, "Resonant nonlinear normal modes. Part I: analytical treatment for structural one-dimensional systems," International Journal of Non-linear Mechanics, vol. 38, no. 6, pp. 851-872, 2003.

[31] A. H. Nayfeh and D. T. Mook, Nonlinear Oscillations, Willey, New York, NY, USA, 1979.

[32] A. H. Nayfeh and B. Balachandran, Applied Nonlinear Dynamics, Wiley-Interscience, New York, NY, USA, 1994. 